\title{
Los depósitos oxfordiano-kimmeridgianos de la Formación Lotena: nuevas perspectivas en la estratigrafía del Jurásico Tardío de la Cuenca Neuquina, Argentina
}

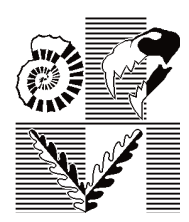

\author{
Daniela E. OLIVERA1, Marcelo A. MARTÍNEZ2, Carlos ZAVALA2,3 y Sara C. BALLENT4
}

\begin{abstract}
THE OXFORDIAN-KIMMERIDGIAN DEPOSITS OF THE LOTENA FORMATION: NEW PERSPECTIVES ON THE LATE JURASSIC STRATIGRAPHY OF THE NEUQUÉN BASIN, ARGENTINA. New micropaleontological and stratigraphical data of the Lotena Formation at Barda Norte locality suggest that the Picún Leufú sub-basin was connected to open marine conditions at least until latest Oxfordian- early Kimmeridgian. These data reinforce the hypothesis of a temporary physical disconnection between the Picún Leufú sub-basin and the Neuquén Basin. The transitional characteristics of the palynological assemblages containing taxa recognized in Argentina since the Late Jurassic and the presence of sporomorphs belonging to the Stereisporites pandoi Zone (late Oxfordian) suggest a latest Oxfordian - early Kimmeridgian age for the analyzed deposits. According to the micropaleontological and stratigraphical analysis, the age of the Lotena Formation in the Picún Leufú sub-basin could span from the middle Callovian to the late Oxfordian-early Kimmeridgian. The microfossils (palynomorphs and foraminifers) suggest a moderate energy marginal-marine environment, located close to the terrigenous source area, and in which salinity varied with changes in freshwater influx.

Resumen. Nuevos datos micropaleontológicos y estratigráficos de la Formación Lotena en la localidad de Barda Norte, localizada en la Subcuenca de Picún Leufú, sugieren que en este sector de la Cuenca Neuquina la conexión marina abierta habría continuado al menos hasta fines del OxfordianoKimmeridgiano. Estos datos refuerzan la hipótesis de una desconexión física temporaria entre la Subcuenca de Picún Leufú y la Cuenca Neuquina. Las características marcadamente transicionales de la asociación palinoflorística identificada en la cual se reconocen taxones presentes en nuestro país a partir del Jurásico Tardío junto con el registro de esporomorfos pertenecientes a la Zona de Stereisporites pandoi (Oxfordiano tardío), sugieren una edad comprendida entre el Oxfordiano tardío y el Kimmeridgiano temprano. En base al análisis micropaleontológico y estratigráfico, se estima que la edad de la Formación Lotena en la Subcuenca de Picún Leufú abarcaría desde el Caloviano medio hasta el Oxfordiano tardíoKimmeridgiano temprano. Los microfósiles (palinomorfos y foraminíferos) permiten inferir un ambiente marino marginal de moderada energía, con cambios en la salinidad relacionados al influjo de agua dulce.
\end{abstract}

Key words. Stratigraphy. Micropaleontology. Palynofacies. Late Jurassic. Neuquén Basin. Argentina.

Palabras clave. Estratigrafía. Micropaleontología. Palinofacies. Jurásico Tardío. Cuenca Neuquina. Argentina

\section{Introducción}

Hasta el presente, los depósitos clásticos oxfordianos de la Cuenca Neuquina habían sido reconocidos únicamente al sur del Cordón de la Piedra Santa, donde fueron asignados a la Formación Fortín $1^{\circ}$ de

\footnotetext{
1Universidad Nacional del Sur, Departamento de Geología, San Juan 670, 8000 Bahía Blanca. danielaolivera2000@yahoo.com.ar ${ }^{2}$ CONICET-INGEOSUR. Universidad Nacional del Sur, Departamento de Geología, San Juan 670, 8000, Bahía Blanca, Argentina.martinez@criba.edu.ar

3GCS Argentina. Haití 123, 8000 Bahía Blanca. czavala@gcsargentina.com

${ }^{4}$ CONICET-División Paleontología Invertebrados, Museo de La Plata. Paseo del Bosque s/n, 1900 La Plata, Argentina.

sballent@fcnym.unlp.edu.ar
}

(c)Asociación Paleontológica Argentina
Mayo (Gulisano et al., 1984). Relevamientos recientes efectuados en el cañón del Arroyo Picún Leufú, localidad Barda Norte, permitieron identificar nuevos afloramientos pertenecientes a los grupos Lotena y Mendoza. La presente contribución constituye un aporte al conocimiento de la Formación Lotena, ya que la integración de datos desde un punto de vista estratigráfico-micropaleontológico permite por primera vez determinar el rango temporal de esta unidad en el sector sur de la Cuenca Neuquina. De esta manera la Formación Lotena podría extenderse a edades tan jóvenes como el Oxfordiano tardíoKimmeridgiano temprano. Nuevos estudios permitirán validar hipótesis previamente establecidas referidas a una posible desconexión física entre la Cuenca Neuquina y la Subcuenca Picún Leufú durante el Bajociano tardío - Kimmeridgiano temprano (Zavala AMGHB2-0002-7014/10\$00.00+.50 


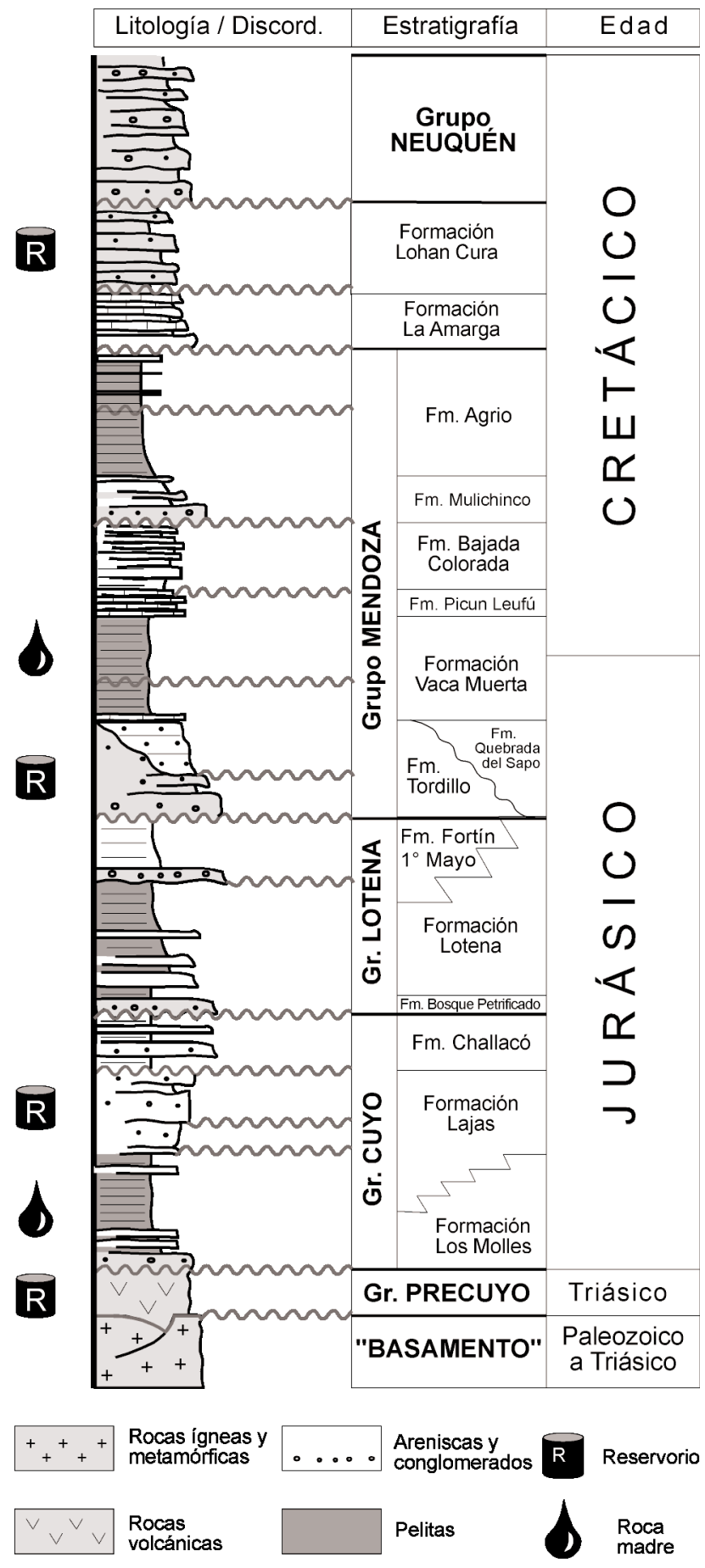

Figura 1. Columna estratigráfica de la Cuenca Neuquina válida para el área de estudio/ stratigraphical column of the Neuquén Basin valid for the study area.

y González, 2001), abriendo de esta manera nuevas líneas de investigación al respecto.

\section{Marco geológico regional y localidad de estu- dio}

La Cuenca Neuquina ha sido definida como una AMEGHINIANA 47 (4), 2010 cuenca de retroarco, desarrollada sobre corteza continental, y originada por el colapso termo-tectónico detrás de un arco magmático estacionario durante el Triásico Tardío (Mpodozis y Ramos, 1989). El relleno sedimentario tuvo lugar principalmente durante el Jurásico y Cretácico, con la acumulación de una sucesión predominantemente clástica de unos $7.000 \mathrm{~m}$ (figura 1), en la cual pueden reconocerse tres etapas principales. La primera etapa (Triásico Tardío - Jurásico Temprano) se caracteriza por la acumulación de materiales volcánicos y volcaniclásticos (Grupo Precuyo) los que presentan espesores sumamente variables y una distribución areal controlada por la presencia de hemigrábenes (Gulisano, 1981; Gulisano et al., 1984). La segunda etapa (Jurásico Temprano a Tardío) se integra por depósitos continentales y marinos progradantes, de naturaleza dominantemente clástica (grupos Cuyo y Lotena), acumulados sobre un relieve irregular controlado por actividad tectónica. Durante esta etapa se produjeron importantes cambios tectónicos y del nivel del mar, provocando un marcado control en las facies y geometría de las unidades correspondientes. La tercera etapa (Jurásico Tardío - Cretácico Tardío) se caracteriza por la acumulación de una potente sucesión marina y continental (grupos Mendoza, Rayoso y Neuquén). Estos últimos depósitos presentan la mayor distribución areal en la cuenca, y sus espesores en general varían de un modo más regular. Los cambios en el nivel del mar y en menor medida la actividad tectónica controlaron el desarrollo de los ciclos internos. Para más detalles sobre la estratigrafía de la Cuenca Neuquina el lector es referido a los trabajos de síntesis de Gulisano et al. (1984), Legarreta y Gulisano (1989), Gulisano y Gutierrez Pleimling (1995) y Legarreta y Uliana (1999).

Los depósitos del Oxfordiano en la Cuenca Neuquina están representados en su mayoría por unidades carbonáticas y evaporíticas asignadas a las formaciones La Manga (Groeber, 1951) y Auquilco (Weaver, 1931; Groeber, 1946) respectivamente, las que han sido vinculadas a un amplio episodio regresivo afectando diversas subcuencas del margen andino (ver síntesis en Vicente, 2006). En el sector sur de la Cuenca Neuquina (Subcuenca Picún Leufú), al sur de la denominada "Dorsal de Huincul", estos depósitos carbonáticos y evaporíticos no han sido reconocidos hasta el presente, por lo que diversos autores (Legarreta y Gulisano, 1989, entre otros) sugieren un importante episodio erosivo, el cual habría truncado los depósitos oxfordianos. No obstante Gulisano et al. (1984) reconocieron localmente una unidad clástica ubicada al sur del Cordón de la Piedra Santa, la cual se localiza en posición estratigráfica entre las formaciones Lotena 
(Caloviano medio) y Tordillo (Kimmeridgiano). Estos autores denominaron a esta unidad Formación Fortín $1^{\circ}$ de Mayo, indicando que la misma podría ser un equivalente temporal de la Formación Auquilco (Oxfordiano tardío). La Formación Fortín $1^{\circ}$ de Mayo está compuesta por unos 60 metros de depósitos continentales. Estos están integrados por conglomerados redepositados, sucedidos por pelitas rojas, y fueron acumulados posiblemente en una cuña sinorogénica localizada al sur de la estructura positiva responsable del levantamiento temprano del Cordón de la Piedra Santa. Estos movimientos intra-oxfordianos son asimismo evidentes en la Sierra de la Vaca Muerta (Anticlinal Covunco y Cerro Irigoyen, Zavala, 2005), los cuales resultan en una dislocación tectónica y erosión de la Formación La Manga, y en el desarrollo de brechas de chert y una importante discordancia erosiva en el contacto de esta con la Formación Auquilco.

Hasta el presente, los depósitos clásticos oxfordianos habían sido identificados únicamente al sur del Cordón de la Piedra Santa (Gulisano et al., 1984). Relevamientos recientes efectuados al sur de la Laguna Blanca, en el cañón del Arroyo Picún Leufú, permitieron reconocer nuevos afloramientos de las formaciones Tordillo y Vaca Muerta. En la localidad de estudio, denominada Barda Norte (figura 2), se disponen 64 metros de conglomerados y areniscas gruesas asignados a la Formación Tordillo, los cuales son sucedidos por más de 120 metros de pelitas negras y margas fosilíferas pertenecientes a los tramos basales de la Formación Vaca Muerta.

Debajo de los conglomerados de la Formación Tordillo se disponen 247 metros de depósitos clásticos sin base expuesta, los cuales fueron asignados temporariamente (Zavala et al., 2008) a la Formación Lotena (figura 2). Estos depósitos están integrados por pelitas grises, con intercalaciones de cuerpos de areniscas finas a conglomerádicas. Los intervalos arenosos constituyen cuerpos tabulares con estratificación tipo hummocky y, localmente, con evidencias de retrabajo por mareas en el techo. Los intervalos clásticos más gruesos componen canales de bajo encauzamiento, con formas tractivas que evidencian paleocorrientes hacia el norte, noreste y noroeste. De este modo, el análisis de facies indica un medio marino de plataforma, afectado ocasionalmente por flujos gravitatorios y modificado por mareas.

\section{Materiales y métodos}

Durante el relevamiento de la sección Barda Norte se tomaron 9 muestras con el fin de analizar su contenido micropaleontológico. El muestreo se realizó considerando las litologías con mayor potencial para el estudio de microfósiles y abarcando en su totalidad el espesor de la unidad considerada. De las muestras analizadas, sólo 2 contienen palinomorfos, lo que permitió realizar recuentos estadísticos (con un mínimo de 250 palinomorfos en cada recuento), siendo consideradas aquí muestras fértiles. En otras 3 el contenido de palinomorfos fue demasiado escaso para permitir este tipo de recuento por lo que se consideraron como muestras con presencias de diferentes formas. La extracción físico-química de la materia orgánica sedimentaria presente en los niveles analizados se efectuó sometiendo el material al ataque con ácido clorhídrico y fluorhídrico. A partir de este residuo se realizaron preparados para estudiar el contenido orgánico palinológico total (palinofacies). Los preparados definitivos se montaron en glicerina-gelatina y el residuo palinológico fue preservado agregando solución de formol. Las muestras palinológicas están depositadas en el Laboratorio de Palinología de la Universidad Nacional del Sur, con las siglas UNSP-BN (Barda Norte). Las coordenadas corresponden a la reglilla England Finder. El estudio palinológico se realizó mediante técnicas de microscopía de luz transmitida, utilizando microscopios Olympus BX40 y Nikon Eclipse 80i. El análisis palinofacial se realizó en base al reconocimiento de los distintos tipos de materia orgánica palinológica (aquí denominado MP) presente según Tyson (1995): palinomorfos, fitoclastos translúcidos, fitoclastos opacos y amorfo. En las figuras 4 y 5 se muestran palinomorfos seleccionados y características de las palinofacies. Las palinofacies se caracterizaron cuantitativamente mediante el recuento de 500 partículas de tamaño superior a los $10 \mu \mathrm{m}$, y se obtuvieron las proporciones relativas de los distintos constituyentes de MP. En la figura 8 se muestran las tendencias próximo-distales que se interpretan para la Palinofacies tipo 2, teniendo en cuenta diversos parámetros palinofaciales seleccionados; si bien la apertura de las cuñas se relaciona con los porcentajes observados, las mismas se encuentran fuera de escala, siendo su representación meramente ilustrativa. En cuanto a los microfósiles calcáreos (foraminíferos) se registró su presencia en 3 muestras. Se procesaron utilizando agua y agua oxigenada (10\%) durante aproximadamente 24 horas y posteriormente lavadas bajo tamiz de abertura $63 \mu \mathrm{m}$ y secadas en estufa a aproximadamente $30^{\circ} \mathrm{C}$. En las descripciones sistemáticas para foraminíferos se ha utilizado la clasificación propuesta por Loeblich y Tappan (1988). En las dimensiones, DM significa diámetro mayor de la conchilla. El material de foraminíferos (ilustrado en la figura 6) queda depositado en la División Paleontología Invertebrados (sección Micropaleontología) del Museo de La Plata, bajo la sigla y numeración MLP-Mi 441 a 448. 

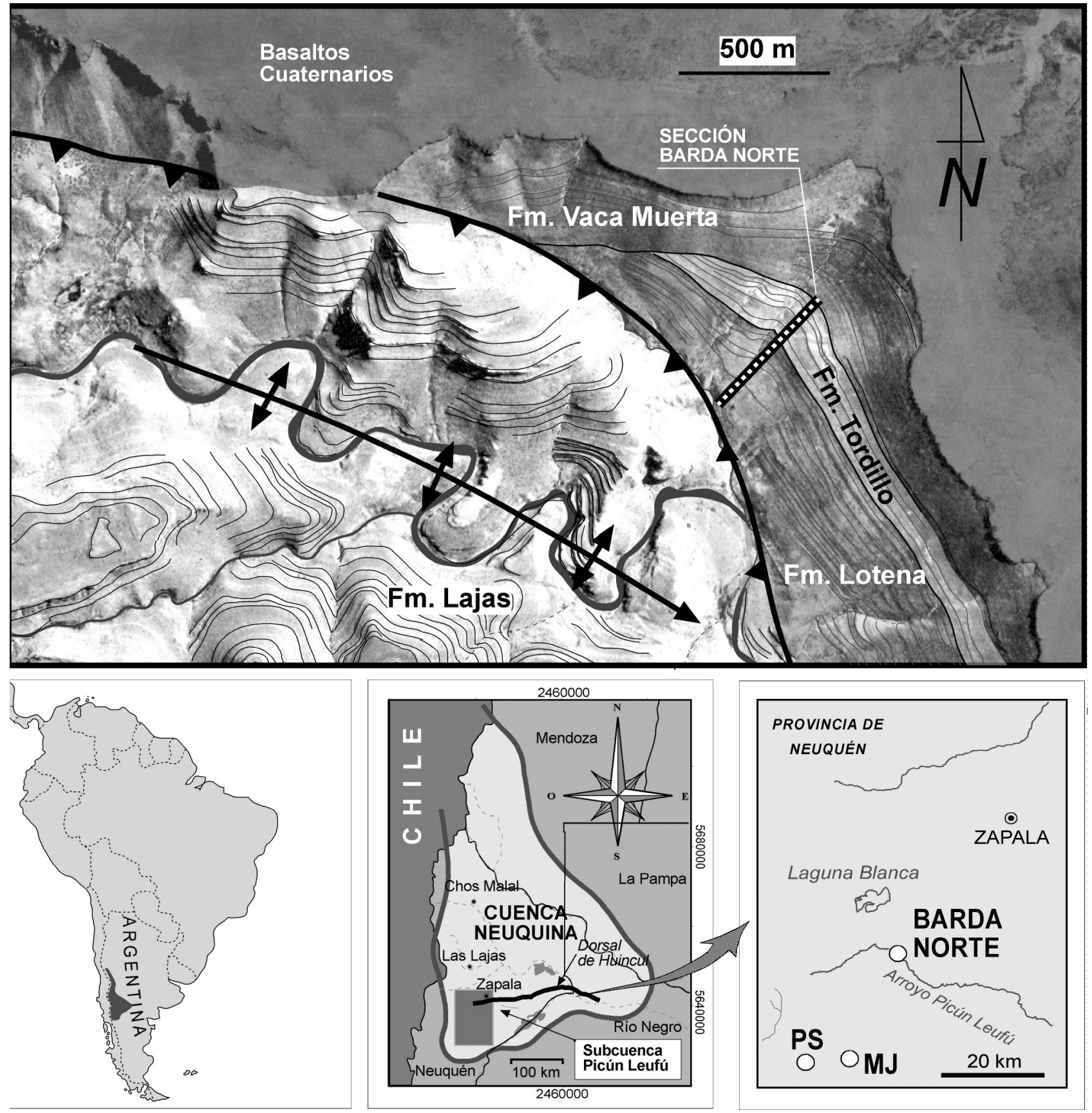

Figura 2. Mapa de América del Sur mostrando la Cuenca Neuquina, la Subcuenca de Picún Leufú y ubicación de la localidad de estudio / map of South America, showing the Neuquén Basin, the Picún Leufú Sub-basin and location of the studied locality. PS: Cordón de la Piedra Santa, MJ: Estancia María Juana.

\section{Paleontología sistemática}

A continuación se describen o comentan aquellas formas con asignación abierta y/o primeras menciones.

\section{Palinomorfos (D. Olivera y M. Martínez)}

\section{Esporas trilete}

Género Matonisporites Couper 1958 AMEGHINIANA 47 (4), 2010
Especie tipo. Matonisporites phlebopteroides Couper 1958.

\section{Matonisporites sp.}

Figura 4.1

Descripción. Espora trilete de contorno triangular y ángulos redondeados. Lados rectos a levemente convexos. Los rayos de lesura se encuentran rodeados por un engrosamiento de la exina de hasta $2 \mu \mathrm{m}$. La cara proximal presenta pequeñas punctuaciones dispuestas en hileras paralelas a los rayos de lesura. La 


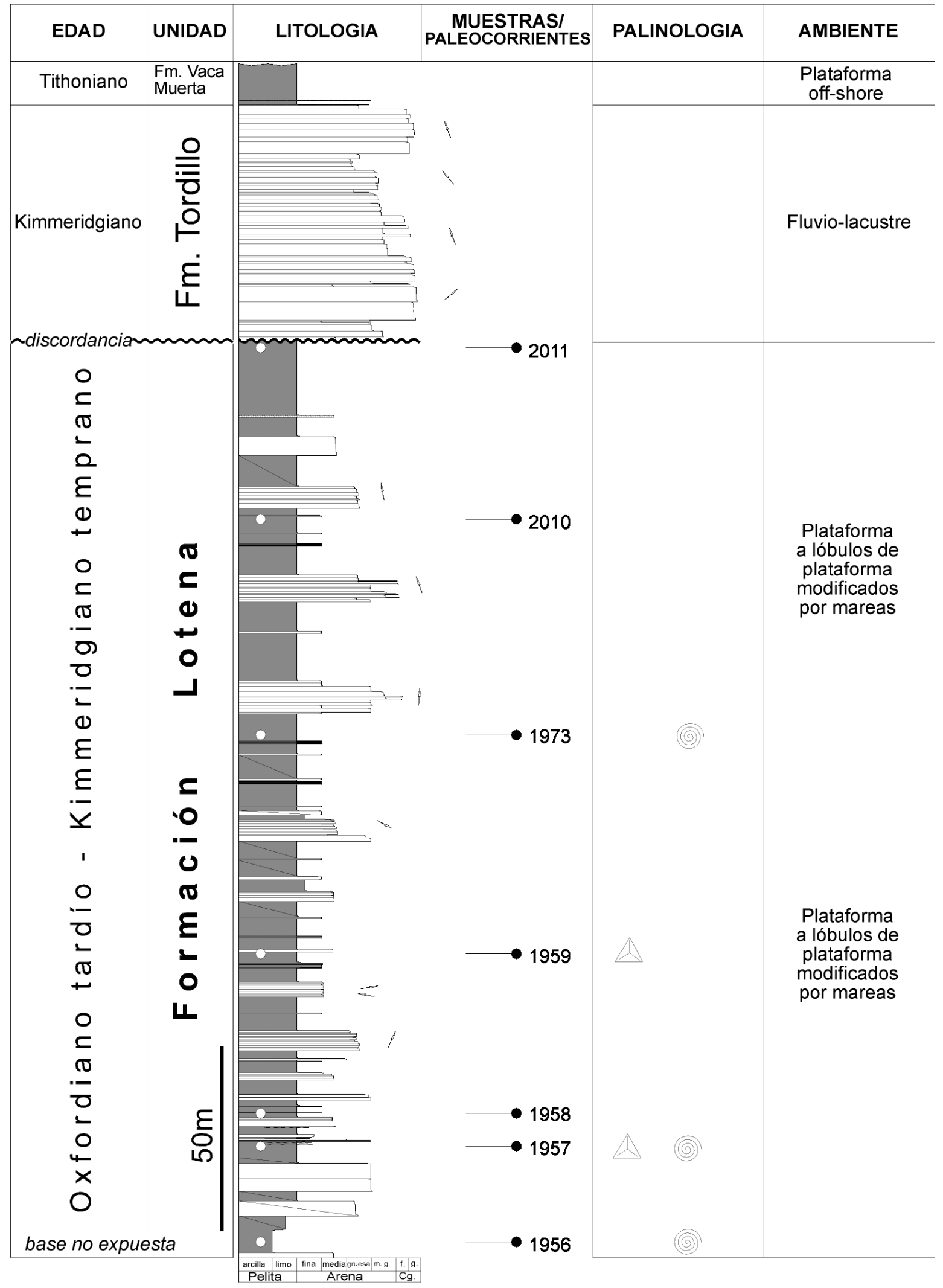

\section{Palinomorfos ((0) Foraminiferos}

Figura 3. Sección Barda Norte con la distribución de las muestras micropaleontológicas / Barda Norte section with the distribution of micropaleontological samples. 
exina se presenta engrosada en los ápices formando valvas que varían entre 2,4 y $3 \mu \mathrm{m}$.

Dimensiones. Diámetro ecuatorial máximo (1 ejemplar): $28 \mu \mathrm{m}$.; espesor de la exina: 1,2 $\mu \mathrm{m}$

Material estudiado. UNSP BN 1959c: E36/4.

Observaciones. El presente material muestra similitud con M. cooksonii Dettmann, taxón presente en la Zona de Oppel de Murospora florida Filatoff (1975), modificada por Helby et al. (1987), de la Cuenca de Perth (Australia). Se diferencia del mismo porque carece de la lesura sinuosa que caracteriza a esta especie y es de menor tamaño que el ejemplar descripto por Dettmann (1963). Asimismo se observa una clara similitud con el ejemplar ilustrado por Filatoff, (1975) como M. crassiangulatus (Balme) Dettmann (lámina 11, fig.12) ampliamente registrado para el Lias tardío al Jurásico Medio, del cual difiere por la presencia de micropunctuaciones en la cara proximal.

\section{Género Leiotriletes (Naumova) Potonié y Kremp 1954}

Especie tipo. Leiotriletes sphaerotriangulus (Loose) Potonié y Kremp 1954.

\section{Leiotriletes sp.}

Figura 4.2

Descripción. Espora trilete de contorno ecuatorial subtriangular, con ápices fuertemente redondeados y lados levemente convexos. Los rayos de lesura, 2/3 a 3/4 del radio de la espora, se encuentran rodeados por un engrosamiento de la exina de 2 a $3 \mu \mathrm{m} .$, Exina homogéneamente punctuada, el diámetro de las punctuaciones es en promedio de $1 \mu \mathrm{m}$, observándose algunas más escasas de hasta $1,5 \mu \mathrm{m}$.

Dimensiones. Diámetro ecuatorial máximo (1 ejemplar): $40 \mu \mathrm{m}$.; espesor de la exina: $1 \mu \mathrm{m}$.

Material estudiado. UNSP BN 1959c: J38/1.

Observaciones. Si bien muestra similitud con el ejemplar mencionado por Archangelsky y Llorens (2003) como Leiotriletes cf. regularis en cuanto a la dimensión, ámbito y espesor de la exina, difiere del mismo por la ornamentación. El presente ejemplar posee un punctuado, a diferencia de la exina lisa a escabrada del material descripto por estos autores.

\section{Género Varirugosisporites Döring 1965}

Especie tipo. Varirugosisporites perverrucatus (Couper) Döring 1965.

AMEGHINIANA 47 (4), 2010

\section{cf. Varirugosisporites mutabilis Döring 1965}

Figura 4.6

1998 Varirugosisporites mutabilis (Döring); Abbink: 162, Lámina II, d, e.

Descripción. Espora trilete de contorno triangular, lados rectos a levemente cóncavos y ápices redondeados. Los rayos de lesura representan entre 2/3 a 4/5 del radio de la espora. La exina se encuentra ornamentada por gránulos y verrugas que varían entre elementos de 1,5 $\mu$ m y $7 \mu \mathrm{m}$ de diámetro en el contorno ecuatorial, llegando a verrugas elongadas hasta de $9 \mu \mathrm{m}$ en torno a la marca trilete donde se concentran los elementos mayores de la ornamentación. El alto de de los gránulos y verrugas varía entre 1 y $1,5 \mu \mathrm{m}$.

Dimensiones. Diámetro ecuatorial máximo (1 ejemplar): $44 \mu \mathrm{m}$, espesor de la exina: $1 \mu \mathrm{m}$.

Material estudiado. UNSP BN 1957c: M14/3.

Observaciones. Los registros mundiales de este taxón comienzan en el Jurásico Temprano tardío (Reyre, 1973) y se extienden hasta el Cretácico Temprano, Valanginiano (Dörhöfer, 1977).

Siguiendo la Zonación Estándar Boreal de amonites, Abbink (1998) registra su presencia en la Zona de lamberti (Caloviano tardío tardío) y en la Zona de densiplicatum (Oxfordiano medio temprano). Tanto el presente ejemplar como el ilustrado por Abbink (1998, Lámina 2 fig. d, e), no se ajustan perfectamente a las características descriptas originalmente para este taxón por Döring (1965). Por este motivo se ha preferido comparar y no asignar el presente material a este género y especie.

\section{Género Ischyosporites Balme 1957}

Especie tipo. Ischyosporites crateris Balme 1957.

Ischyosporites sp. cf. I. sp. C (en Volkheimer, 1972) Figura 4.7

Dimensiones. Diámetro ecuatorial máximo (1 ejemplar): $46 \mu \mathrm{m}$.

Material estudiado. UNSP BN 1957c: G61.

Observaciones. Se compara con Ischyosporites sp. C descripto por Volkheimer (1972), dado que el material aquí estudiado presenta ámbito triangular, tamaño levemente mayor y valvas más gruesas $(4 \mu \mathrm{m})$. Cabe destacar que presenta también ciertas similitudes con el material asignado a Ischyosporites marburgensis por Filatoff (1975) de la cual difiere por carecer del margo punctuado que presenta esta especie. 
Género Duplexisporites Deák emend. Playford y Dettmann 1965

Especie tipo. Duplexisporites generalis Deák 1962.

\section{Duplexisporites sp.}

Figura 4.15

Descripción. Espora trilete cingulada de contorno ecuatorial subtriangular. Las ramas de la lesura son simples, rectas a levemente sinuosas y se bifurcan cuando alcanzan el margen interno del cíngulo. La cara proximal, escabrada a microgranulada, presenta un muro escasamente definido, dispuesto tangencialmente al borde ecuatorial. La escultura de la cara distal está representada por muros que forman una espiral discontinua cuyo centro se ubica en el polo distal, sin alcanzar el cíngulo y por gránulos de hasta 2 $\mu \mathrm{m}$ de diámetro y escaso relieve (hasta $1 \mu \mathrm{m}$ de alto) dispuestos homogéneamente en toda la superficie.

Dimensiones. Diámetro ecuatorial máximo (1 ejemplar): $56 \mu \mathrm{m}$; ancho del cíngulo: 2-4 $\mu \mathrm{m}$; ancho de los muros: 3-7 $\mu \mathrm{m}$.

Material estudiado. UNSP BN 1959c: N21.

Observaciones. Muestra similitud con Duplexisporites? sp. B de Volkheimer y Quattrocchio (1975). Sin embargo, el presente ejemplar es de tamaño mayor y presenta un muro en la cara proximal, el cual no se distingue en el mencionado por estos autores.

\section{Género Gemmatriletes Pierce 1961}

Especie tipo. Gemmatriletes morulus Pierce 1961.

\section{Gemmatriletes sp. cf. G. covuncoensis Volkheimer y Moroni 1981 Figura 4.11}

Descripción. Espora trilete de contorno ecuatorial subcircular a levemente subtriangular. Rayos de lesura simples, rectos que llegan casi al ecuador, la relación de los rayos de lesura con el radio de la espora es mayor o igual a 4/5. Exina densamente ornamentada por gemas y clavas con un marcado predominio de las primeras. Sobre el contorno ecuatorial se contaron 48 elementos ornamentales.

Dimensiones. Diámetro ecuatorial máximo incluyendo la ornamentación (1 ejemplar): $34 \mu \mathrm{m}$; ancho de las gemas: 1- $4 \mu \mathrm{m}$; largo de las gemas: hasta $3 \mu \mathrm{m}$.

Material estudiado. UNSP BN 1957c: L59/4.

Observaciones. El cf. hace referencia al menor tamaño de las gemas en el ejemplar aquí estudiado en comparación con el material descripto por Volkheimer y Moroni (1981). El género Gemmatriletes Pierce ha sido ampliamente citado a nivel mundial a partir del Cretácico Temprano. En nuestro país fue registrado entre otros por Volkheimer y Moroni (1981) durante el Jurásico Tardío temprano y por Volkheimer y Zavattieri (1991) y Volkheimer y Papu (1993) para depósitos triásicos.

\section{Género Klukisporites Couper 1958}

Especie tipo. Klukisporites variegatus Couper 1958.

\section{Klukisporites sp. cf. K. scaberis (Cookson y Dettmann) Dettmann 1963 \\ Figura 4.12}

Descripción. Espora trilete de ámbito subredondeado. Rayos de la lesura rectos extendidos casi hasta el ecuador, acompañados por mayor concentración de ornamentos sin llegar a formar verdaderos labios. En la cara distal, muros de hasta $3 \mu \mathrm{m}$ de ancho describen un retículo de lúminas irregulares. Pequeños gránulos y verrugas se disponen en ambas caras, confinados a las bases de la lúminas en la cara distal y en torno a la lesura en la cara proximal.

Dimensiones. Diámetro ecuatorial máximo (1 ejemplar): $54 \mu \mathrm{m}$.

Material estudiado. UNSP BN 1957c: Q43/2.

Observaciones. Se compara y no se asigna a Klukisporites scaberis dado que no se observa claramente el desarrollo de gruesos labios característicos de esta especie, la cual ha sido ampliamente citada durante el Mesozoico tardío de Australia, siendo un elemento muy común de la Zona de Oppel de Murospora florida (Caloviano medio tardío Kimmeridgiano) (Filatoff, 1975), modificada por Helby et al. (1987).

\section{Género Osmundacidites Couper 1953}

Especie tipo. Osmundacidites wellmanii Couper 1953.

Osmundacidites sp. cf. O. wellmanii Couper 1953 Figura 4.13

2005 Osmundacidites cf. O. wellmanii (Couper); Archangelsky y Llorens: 322, figura $3 b$.

Descripción. Espora trilete de contorno circular. Lesura recta con labios de hasta $1 \mu \mathrm{m}$ de espesor, ornamentados con microgránulos al igual que la cara proximal y distal. Los elementos esculturales se encuentran homogéneamente distribuidos en ambas caras. 
Dimensiones. Diámetro ecuatorial máximo (1 ejemplar): $49 \mu \mathrm{m}$.

Material estudiado. UNSP BN 1957c: N58/1.

Observaciones. Difiere de Osmundacidites wellmanii por la presencia de labios y ornamentos más pequeños y menos diversos.

Gen. et sp. indet.

Figura 4.14

Descripción. Espora trilete de ámbito circular, rayos de la lesura alcanzando casi el ecuador, la relación de los rayos de lesura con el radio de la espora es mayor o igual a 7/8 . Ornamentación representada principalmente por espinas, de hasta $5 \mu \mathrm{m}$ de longitud y hasta $1 \mu \mathrm{m}$ de diámetro. De manera subordinada se identifican báculas y espinas truncadas. Cara distal fuerte y homogéneamente ornamentada y cara proximal con elementos esculturales dispuestos más espaciadamente.

Dimensiones. Diámetro ecuatorial máximo con ornamentación (1 ejemplar): $33 \mu \mathrm{m}$.

Material estudiado. UNSP BN 1957c: X59/2.

Observaciones. Rojo y Zavattieri (2005), revisaron dos géneros afines, Ceratosporites y Neoraistrickia. Se discuten sus diferencias y se remarca la ornamentación de la cara proximal como principal criterio de distinción: exina proximal lisa en Ceratosporites y ornamentada en Neoraistrickia siguiendo el criterio propuesto por de Jersey (1971). El presente ejemplar presenta ámbito redondeado y ornamentación esencialmente equinada como en Ceratosporites y elementos esculturales tanto en la cara distal como en la proximal (con mayor espaciamiento en esta última), como en Neoraistrickia. Difiere de Ceratosporites por la marcada presencia de ornamentación en la cara proximal y de Neoraistrickia por el ámbito circular en lugar de triangular redondeado y ornamentación esencialmente equinada en lugar de baculada.

Comparaciones. Muestra cierta similitud con Neoraistrickia sp. (Rojo y Zavattieri, 2005, Fig. 4M), sin embargo en esta última, las áreas interradiales de la cara proximal poseen ornamentación reducida a gránulos o conos hasta una exina lisa en algunos ejemplares. Dado el carácter transicional y la escasez del presente material (un único ejemplar) se mantiene su asignación abierta.

\section{Granos de polen}

Género Balmeiopsis Archangelsky 1977

AMEGHINIANA 47 (4), 2010
Especie tipo. Balmeiopsis limbatus (Balme) Archangelsky 1977.

\section{Balmeiopsis limbatus Archangelsky 1977}

Figura 4.23

Dimensiones. Diámetro mayor (2 ejemplares): 72-89 $\mu \mathrm{m}$; espesor de la exina 2-3 $\mu \mathrm{m}$.

Material estudiado. UNSP BN 1959c: F23, F38.

Observaciones. Este taxón ha sido reconocido desde el Jurásico Tardío en el norte de Africa (Reyre en Volkheimer y Prámparo, 1993) hasta el Albiano en Australia y el Albiano medio en Canadá (Rich, et al., 1989; Zippi y Bajc, 1990; Alley et al., 1996). El registro del mismo durante el Cretácico Temprano de la Argentina es amplio, habiendo sido citado por diversos autores entre los que puede mencionarse a Archangelsky (1977), Archangelsky y Seiler (1980), Volkheimer y Quattrocchio (1981), Archangelsky et al. (1984), Baldoni y Batten (1991), Volkheimer y Prámparo (1993) y Prámparo (1994). Los registros pre-cretácicos en el país son muy escasos, pudiendo mencionarse a Volkheimer y Prámparo (1993) donde se hace la primer mención de Balmeiopsis limbatus en depósitos correspondientes a la Formación Tordillo (Kimmeridgiano) en la localidad Chacay Melehue. Probablemente estos afloramientos hayan sido erróneamente asignados a la Formación Tordillo y representen facies laterales de la Formación Mulichinco (Prámparo com. pers., 2003). Quattrocchio et al. (2003) citaron este taxón desde el Tithoniano tardío (Formación Quintuco en la localidad Mallín Quemado) hasta el Valanginiano tardío. Constituye ésta la primera mención de Balmeiopsis limbatus para depósitos pre-tithonianos del país.

\section{Quistes de dinoflagelados}

\section{Género Lithodinia Eisenack emend. Gocht 1975}

Especie tipo. Lithodinia jurassica Eisenack emend. Gocht 1975.

Lithodinia deflandrei (Sarjeant) Gocht 1976 Figura 5.1

Dimensiones. Largo (sin el opérculo) (1 ejemplar): 45 $\mu \mathrm{m}$; ancho: $51 \mu \mathrm{m}$.

Material estudiado. UNSP BN 1959c: Q31.

Observaciones. Se diferencia de otras especies del género por la ornamentación parasutural e intratabular consistente en pequeños dentículos unidos ocasionalmente por trabéculas. Constituye la primera mención para la Cuenca Neuquina. 
Género Acanthaulax Sarjeant emend. Sarjeant 1982

Especie tipo. Acanthaulax venusta (Klement) Sarjeant emend. Sarjeant 1982

\section{Acanthaulax downiei (Sarjeant) Sarjeant 1976}

Figura 5.2

Dimensiones. Ancho (1 ejemplar): $31 \mu \mathrm{m}$; largo: 35 $\mu \mathrm{m}$.

Material estudiado. UNSP BN 1959c: H31/2.

Observaciones. Globalmente, este taxón es asignado a estratos oxfordianos, sin embargo en nuestro país Quattrocchio et al. (1996) definieron la zona de Acanthaulax downiei para el Tithoniano temprano a medio. Algunos registros esporádicos pre-tithonianos en la Cuenca Neuquina corresponden a Martínez (2002), quien mencionó Acanthaulax sp. en la Sub-biozona de Klukisporites labiatus y ?Acanthaulax downiei en la Subbiozona de Neoraistrickia truncata, ambas correspondientes al Bajociano temprano de la Formación Lajas.

\section{Foraminíferos (S. Ballent)}

\section{Género Ammodiscus Reuss, 1862}

Especie tipo. Ammodiscus infimus Bornemann, 1874.

\section{Ammodiscus sp.}

Figuras 6.1-8

Descripción. Conchilla aglutinada, pequeña (diámetro promedio $0,250-0,350 \mathrm{~mm}$ ), discoidal, de contorno subelipsoidal a subcircular y periferia redondeada a ligeramente comprimida, formada por un prolóculo, generalmente no distinguible, seguido por una cámara tubular larga con enroscamiento planospiral evoluto dispuesta en 4-5 vueltas. Las vueltas van aumentando su alto a medida que se agregan, siendo la última la más conspicua debido a que la sutura espiral entre ella y la anteúltima vuelta es marcadamente deprimida. Asimismo la última vuelta puede recubrir ligeramente a la vuelta anterior. Abertura terminal al final de la cámara tubular. Pared aglutinada de grano fino y aspecto granular, de material siliciclástico y carbonático de color blanco.

Dimensiones (en $\mathrm{mm}$ ). Ejemplares ilustrados: MLP-Mi 441, DM= 0, 358 (figura 6.1); MLP-Mi 442, $\mathrm{DM}=0,291$ (figura 6.2); MLP-Mi 443, DM=0, 199 (figuras 6.3-4); MLP-Mi 444, DM= 0, 240 (figura 6.5); MLP-Mi 445, DM=0, 266 (figura 6.6); MLP-Mi 446, DM=0, 195 (figura 6.7); MLP-Mi 447, DM=0, 200 (figura 6.8).
Material. 30 conchillas. MLP-Mi 441-448.

Observaciones. El aspecto de la conchilla es variable, desde contorno casi circular, subcircular hasta subelipsoidal elongado, con periferia irregular aparentemente condicionada por la forma de un sustrato duro, lo que estaría indicando que podría tratarse de una forma que vivió adherida. El tamaño de grano que aglutina es generalmente fino, aunque también los hay de tamaño medio, manteniendo siempre el aspecto granular o "áspero". Aparecen también algunas conchillas con una pequeña porción inicial con enroscamiento irregular (streptospiral?) del tipo de Glomospirella Plummer. Ammodiscus sp. es muy similar a $A$. asper (Terquem, 1863) de amplia distribución en el Jurásico Temprano de Europa, América del Norte y norte de África, pero esta especie es de tamaño grande (diámetro mayor a $1 \mathrm{~mm}$ ), bicóncava en vista periférica, con material grueso adherido y aspecto rugoso. Especies jurásicas de tamaño pequeño y que poseen cierta similitud con la presente son $A$. orbis Lalicker (1950) del Grupo Ellis, OxfordianoKimmeridgiano de Montana (Estados Unidos de América), A. francisi Wall, 1960, y A. southeyensis de Wall, 1960, del Caloviano-Oxfordiano de Saskatchewan, Canadá. La primera de ellas, empero, aglutina granos de cuarzo muy finos y cemento ferruginoso y posee la sutura espiral deprimida y muy nítida; A. francisi Wall es muy similar a la presente pero aglutina material arenoso extremadamente fino y su aspecto es vítreo; A. southeyensis Wall, es de tamaño pequeño a medio, y con la última vuelta muy alta. Ammodiscus sp. se diferencia de otras especies jurásicas por sus dimensiones pequeñas y por el tamaño de grano del sedimento aglutinado. Si bien hay una serie de ejemplos referidos a la selectividad que manifiestan los foraminíferos aglutinados en cuanto a los materiales y tamaño de grano para construir su conchilla, no hay que sobreestimar esta capacidad dándole siempre un valor genérico o específico (Boltovskoy, 1965). Está comprobado que, a falta de su "material preferido", ellos utilizan para construir la pared de su conchilla el material que tienen a su disposición en el medio en que viven. Teniendo en cuenta esto y considerando además que los especímenes estudiados en este trabajo exhiben una variabilidad que podría estar relacionada con cambios en las condiciones paleoambientales, se mantiene el taxón en nomenclatura abierta como Ammodiscus sp.

\section{Resultados}

Asociaciones palinológicas

El análisis porcentual de las microfloras muestra AMEGHINIANA 47 (4), 2010 
que las mismas están principalmente constituidas por esporomorfos (granos de polen y esporas), siendo muy escaso el contenido de microplancton marino de pared orgánica (figura 7). El porcentaje de palinomorfos continentales varía entre un 93,7\% y un $96,7 \%$ en tanto que el microplancton marino de pared orgánica fluctúa entre un 3,3 \% y un 6,3\%. Dentro de los granos de polen dominan las cheirolepidiáceas, acompañadas por araucariáceas, podocarpáceas, pteridospermas y el complejo Callialasporites (figura 7). Las esporas se presentan de manera subordinada, con diversidades que no superan los 20 taxones. El microplancton marino de pared orgánica es poco diverso y escaso, predominando acritarcos Acanthomorphitae del complejo Micrhystridium y dinoquistes proximados tales como Acanthaulax downiei, Escharisphaeridia pocockii, Lithodinia deflandrei entre otros (tabla 1 y figura 5).

\section{Análisis palinofacial}

En el presente trabajo se utiliza el término palinofacies como "el cuerpo de roca sedimentaria que contiene asociaciones diferenciadas de materia orgánica palinológica, las cuales reflejan el conjunto de las condiciones ambientales o están asociadas a un rango característico de potencial de generación de hidrocarburos" (Tyson, 1995). En base al análisis de la materia orgánica palinológica total fue posible diferenciar tres tipos de palinofacies. Las mismas se definieron en base a la frecuencia relativa de las cuatro categorías de materia orgánica palinológica (MP): palinomorfos, fitoclastos translúcidos y opacos y materia orgánica amorfa (figuras 5 y 7).

Palinofacies-tipo 1 (P-1). Identificada en las muestras 1956, 1958, 2010 y 2011. Se caracteriza por una alta proporción de fitoclastos translúcidos (75\% a $84 \%$ ). Los fitoclastos opacos representan de un $12 \%$ a un $16 \%$ del total de la MP, la materia orgánica amorfa no excede el $9 \%$ y solo se registra presencia de palinomorfos. Dentro de los fitoclastos translúcidos predominan los no-bioestructurados de color castaño oscuro bien preservados, especialmente los que varían entre 11 y $40 \mu \mathrm{m}$. Dentro de los fitoclastos opacos, tienen mayor representación las tablillas. La materia orgánica amorfa, está dominada por el tipo esponjoso (51\%) y de manera subordinada el tipo granular (35\%) (figuras 5.9 y 7).

Palinofacies-tipo 2 (P-2). Identificada en las muestras 1957 y 1959. Se caracteriza por la presencia de muestras fértiles en palinomorfos, los cuales representan entre un 4 y $5 \%$ del total de la MP. Los fitoclastos translúcidos varían entre 60 y $85 \%$ y los opacos entre 8 y 25\%. El grupo con menor representación corresponde a la materia orgánica amorfa (2-10\%). Dentro de los fitoclastos translúcidos prevalecen los no-bioestructurados bien preservados de color castaño, con morfología elongada de entre 11 y $40 \mu \mathrm{m}$. A diferencia de la P-1, se identificó un pequeño porcentaje de fitoclastos translúcidos bioestructurados (principalmente traqueidas y en menor proporción cutículas). Dentro de los fitoclastos opacos predominan las tablillas de hasta $60 \mu \mathrm{m}$ y dentro de la materia orgánica amorfa tiene mayor representación la de tipo granular (figuras 5.10-13, 7 y 8).

Palinofacies-tipo 3 (P-3). Identificada en la muestra 1973; si bien los fitoclastos translúcidos constituyen el grupo con mayor representación (60\%), a diferencia de las otras dos palinofacies, la materia orgánica amorfa representa el 32\% de la materia orgánica dispersa. El porcentaje de fitoclastos opacos no excede el 7\% y sólo se registra presencia de palinomorfos. Dentro de los fitoclastos translúcidos, predominan los no-bioestructurados de color castaño, bien preservados que no exceden los $40 \mu \mathrm{m}$. Los fitoclastos opacos muestran similares proporciones de tablillas y equidimensionales. Dentro de la materia orgánica amorfa, un $40 \%$ corresponde al tipo granular, un $35 \%$ al tipo esponjoso y el 25\% restante está representado por materia orgánica amorfa indiferenciada (figuras 5.14 y 7 ).

Figura 4. 1, Matonisporites sp., UNSP BN 1959c: E36/4; 2, Leiotriletes sp., UNSP BN 1959c: J38/1; 3, Concavisporites semiangulatus Menéndez 1968, UNSP BN 1957c: X49; 4, Granulatisporites sp. A (en McKellar, 1974), UNSP BN 1957c: V60/1; 5, Granulatisporites sp. C (en Volkheimer, 1972), UNSP BN 1957c: O55/2; 6, cf. Varirugosisporites mutabilis Döring 1965, UNSP BN 1957c: M14/3; 7, Ischyosporites cf. I. sp. C (en Volkheimer, 1972), UNSP BN 1957c: G61; 8, Staplinisporites caminus (Balme) Pocock 1962, UNSP BN 1959c: H23/4; 9, Stereisporites antiquasporites (Wilson y Webster) Dettmann 1963, UNSP BN 1959c: U34/2; 10, Antulsporites saevus (Balme) Archangelsky y Gamerro 1966, UNSP BN 1959c: T35/4; 11, Gemmatriletes sp. cf. G. covuncoensis Volkheimer y Moroni 1981, UNSP BN 1957c: L59/4; 12, Klukisporites sp. cf. K. scaberis (Cookson y Dettmann) Dettmann 1963, UNSP BN 1957c: Q43/2; 13, Osmundacidites sp. cf. O. wellmanii (en Archangelsky y Llorens, 2005), UNSP BN 1957c: O58/1; 14, Gen. et sp. indet., UNSP BN 1957: X59/2); 15, Duplexisporites sp., UNSP BN 1959c: N21; 16, Pilosisporites sp. 2 (en Martínez, Quattrocchio y Sarjeant, 2001), UNSP BN 1959c: G39/2; 17, Klukisporites variegatus Couper 1958, UNSP BN 1957c:M14/3; 18, Klukisporites labiatus (Volkheimer) Baldoni y Archangelsky 1983, UNSP BN 1957c:Q46/1; 19, Interulobites lajensis Martínez 2000, UNSP BN 1959c: L23/1; 20, Callialasporites turbatus (Balme) Schulz 1967, UNSP BN 1959c: L20/2; 21, Callialasporites trilobatus (Balme) Dev 1961, UNSP BN 1957c: E37/4; 22, Microcachryidites castellanosii Menéndez 1968, UNSP BN 1959c: G25/3; 23, Balmeiopsis limbatus Archangelsky 1977, UNSP BN 1959c: F38; 24, Peroaletes rugosus McKellar 1974, UNSP BN 1957c: J44/3. Escala gráfica / scale bar $=10 \mu \mathrm{m}$. 

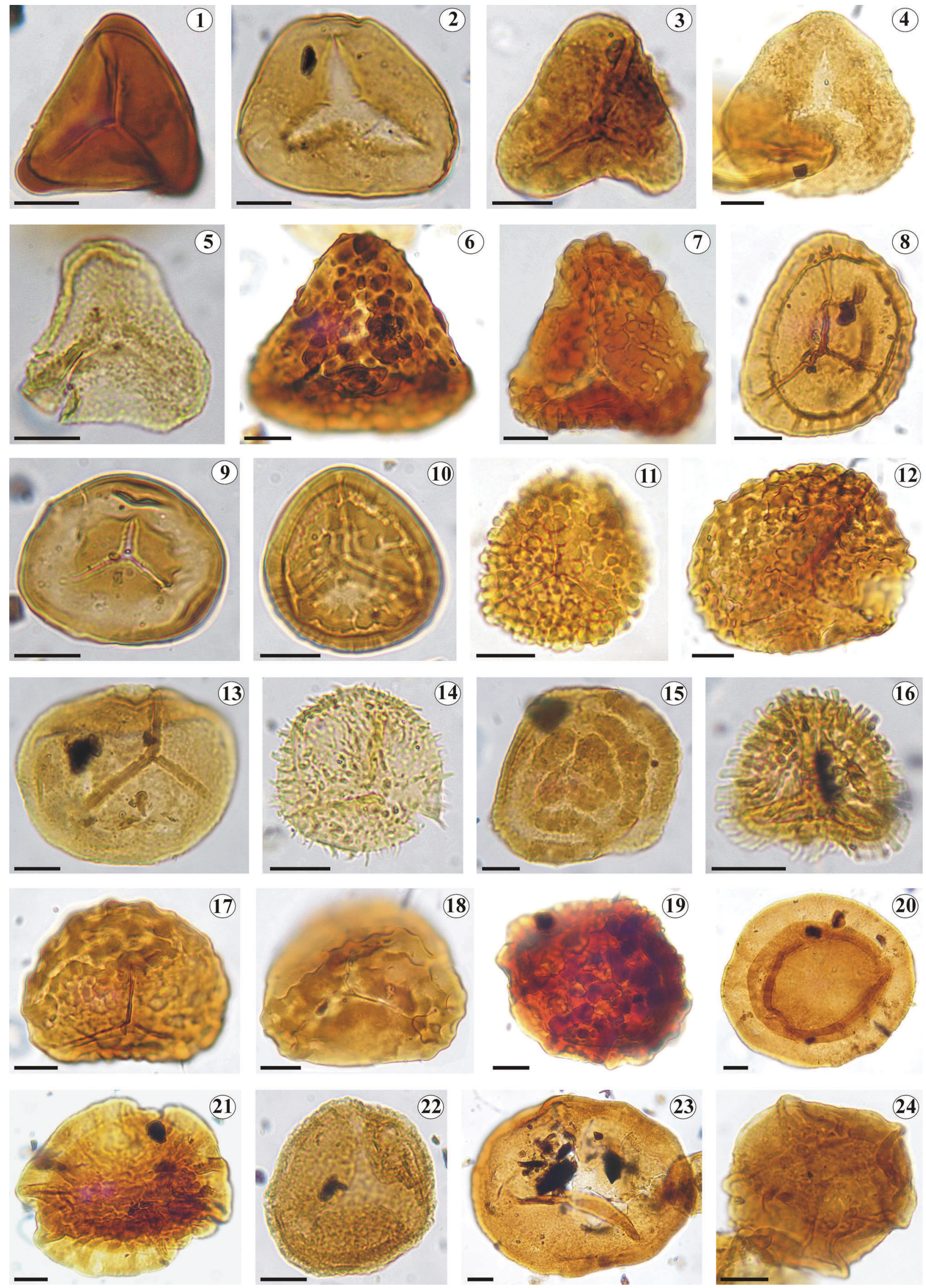

AMEGHINIANA 47 (4), 2010 

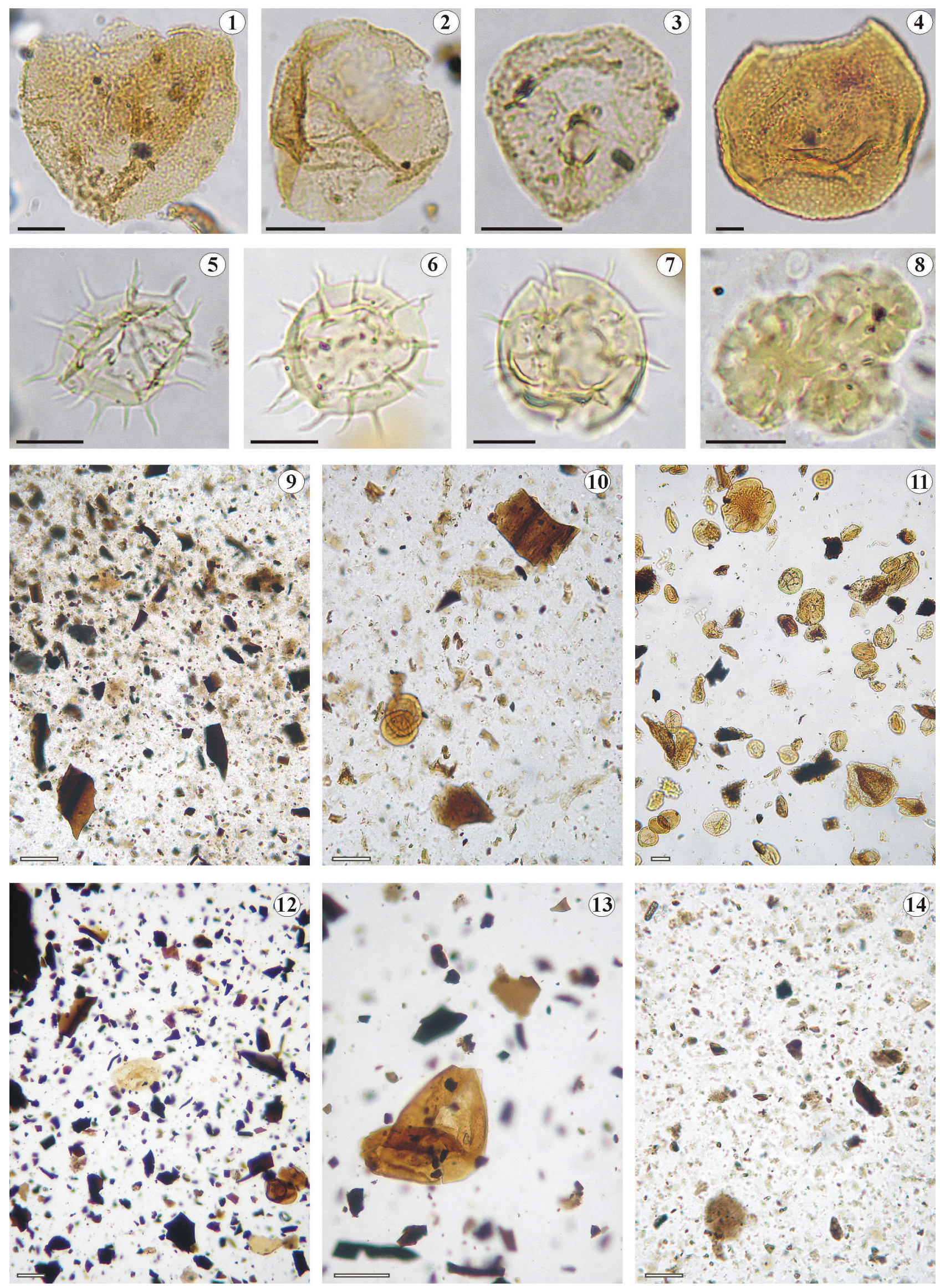

000 v 14 $4 \cos x-3=0$

AMEGHINIANA 47 (4), 2010 


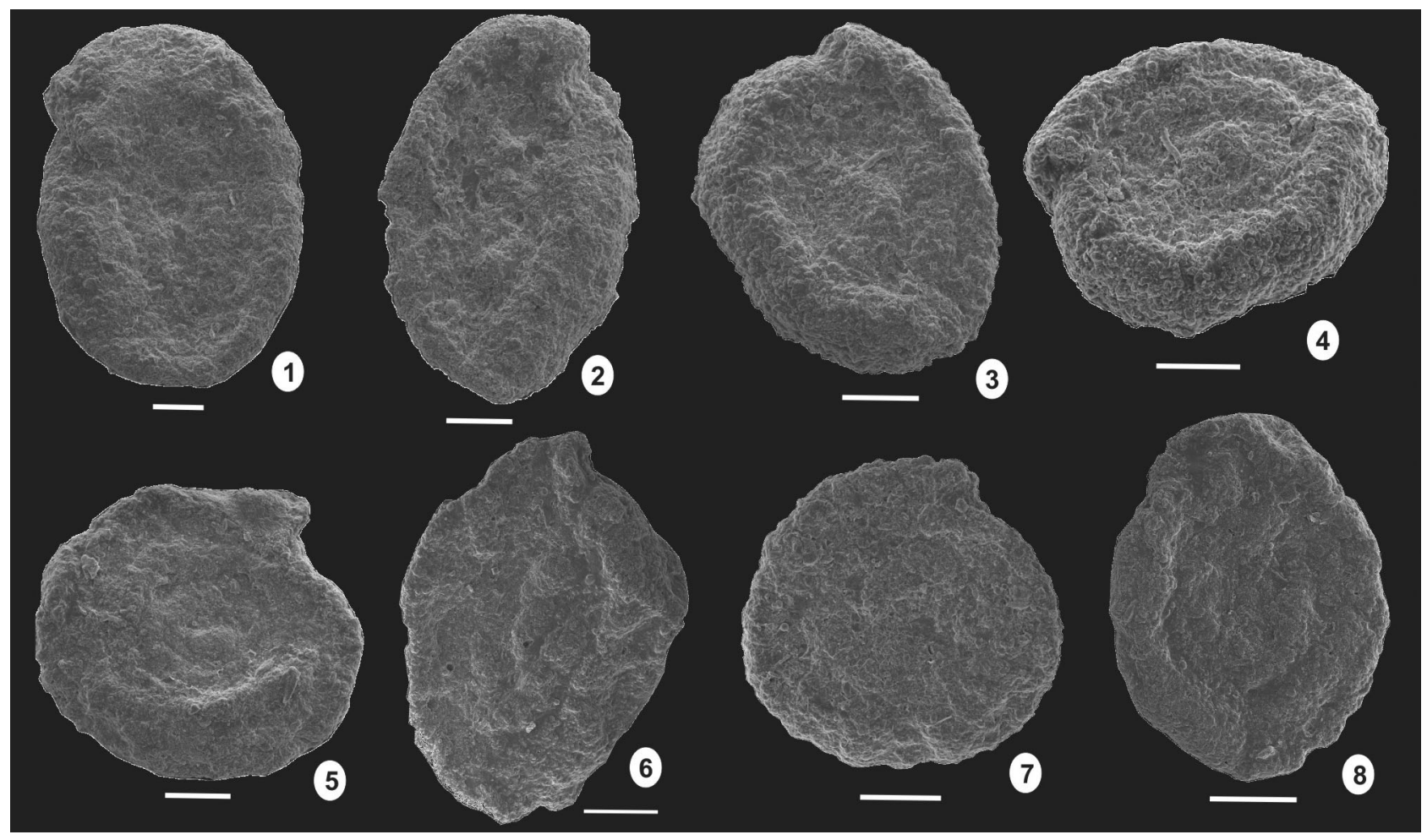

Figura 6. Ammodiscus sp. 1, MLP-Mi 441, muestra / sample 1956, vista lateral / lateral view; 2, MLP-Mi 442, muestra / sample 1956, vista lateral / lateral view; 3-4, MLP-Mi 443, muestra / sample 1956, 3, vista lateral / lateral view; 4, vista apertural / apertural view; 5, MLP-Mi 444, muestra / sample 1957, vista lateral / lateral view; 6, MLP-Mi 445, muestra / sample 1957, vista lateral / lateral view; 7, MLP-Mi 446, muestra / sample 1956, vista lateral / lateral view; 8, MLP-Mi 447, muestra / sample 1957, vista lateral / lateral view. Escala gráfica / Scale bar $=0.050 \mathrm{~mm}$.

\section{Composición de la microfauna de foraminíferos}

La asociación, recuperada en las muestras 1956, 1957 y 1973, se caracteriza por su baja diversidad, ausencia de formas calcáreas, estando presentes, con moderada abundancia, solamente foraminíferos de pared aglutinada asignados a Ammodiscus sp. Se trata de una especie de tamaño pequeño, variable en cuanto al contorno de la conchilla desde formas casi circulares hasta ovoidales elongadas o subelipsiodales y de periferia irregular, característica ésta que podría estar indicando un hábito adherente.

\section{Análisis palinoestratigráfico}

Si bien el contenido de foraminíferos no permite realizar un estudio acerca de la edad precisa de los depósitos portadores de la asociación, el análisis palinológico revela la presencia de algunos taxones cuyo comportamiento en el intervalo estudiado es, al menos, llamativo. Quattrocchio et al. (1996) definieron para el Tithoniano de la Cuenca Neuquina la Zona de Microcachryidites antarcticus que se caracteriza por un significativo incremento de este taxón acompañado por una disminución en la abundancia del complejo Callialasporites, caracterizando de esta

Figura 5. 1, Lithodinia deflandrei (Sarjeant) Gocht 1976, UNSP BN 1959c: Q31; 2, Acanthaulax downiei (Sarjeant) Sarjeant 1976, UNSP BN 1959c: H31/2; 3, Escharisphaeridia pocockii (Sarjeant) Erkmen y Sarjeant 1980, UNSP BN 1959c: E22; 4, Tasmanites sp., UNSP BN 1957c: W57; 5, Michrystridium echinoides Valensi 1953, UNSP BN 1957c: D58; 6, Michrystridium recurvatum forma recurvatum Valensi 1953, UNSP BN 1957c: D58; 7, Michrystridium brevispinosum Sarjeant 1961, UNSP BN 1957c: D57; 8, Botryococcus sp., UNSP BN $1957 \mathrm{~b}$ : C10/3; 9, Palinofacies-tipo 1 (UNSP BN 1956b), caracterizada por una alta proporción de fitoclastos translúcidos castaño oscuro / characterized by a high proportion of dark brown translucent phytoclasts; 10-11, Palinofacies-tipo 2 (UNSP BN 1957), 10, luego de remoción de la fracción mineral / after removal of mineral fraction; 11, se ilustra la alta concentración de esporomorfos luego de aplicar técnicas de separación con líquidos pesados (Cl2Zn, densidad: 1,4) y centrifugados breves / high concentration of sporomorphs can be observed after treatement with heavy liquid techniques (Cl2Zn, density: 1.4) and then briefly centrifuged; 12-13, Palinofacies-tipo 2 (UNSP BN 1959), 12, aspecto general de la palinofacies con alta proporción de fitoclastos translúcidos y presencia de palinomorfos / general aspect of the palynofacies with high proportion of translucent phytoclasts and presence of palynomorphs; 13, detalle de un palinomorfo / detail of a palynomorph (Araucariacites fissus Reiser y Williams 1969); Palinofacies-tipo 3 (UNSP BN 1973) caracterizada por una mayor proporción de materia orgánica amorfa / characterized by a higher proportion of amorphous organic matter. Escala gráfica $=10 \mu \mathrm{m}$, excepto en figuras $9-14=25$ $\mu \mathrm{m} /$ scale bar $=10 \mu \mathrm{m}$, except in figures 9-14 $=25 \mu \mathrm{m}$. 
Tabla 1. Distribución de frecuencias relativas de palinomorfos ordenados según los grupos supragenéricos / distribution of relative frequencies of palynomorphs ordered according to suprageneric groups.

\begin{tabular}{|c|c|c|c|c|c|}
\hline Grupos Supragenéricos & Taxones & \multicolumn{2}{|c|}{1957} & \multicolumn{2}{|c|}{1959} \\
\hline Cheirolepideaceae & $\begin{array}{l}\text { Classopollis classoides (Pflug) Pocock y Jansonius } 1961 \\
\text { Classopollis intrareticulatus Volkheimer } 1972 \\
\text { Classopollis major Groot y Groot } 1962 \\
\text { Classopollis simplex (Danzé, Corsin y Laveine) Reiser y Williams } 1969 \\
\text { Classopollis torosus (Reissinger) Burger } 1965 \\
\text { Classopollis spp. }\end{array}$ & 69,4 & $\begin{array}{l}30,4 \\
5,12 \\
3,14 \\
1,04 \\
5,1 \\
24,6\end{array}$ & 52,1 & $\begin{array}{l}23 \\
0,7 \\
0,7 \\
0,7 \\
3,8 \\
23,2\end{array}$ \\
\hline $\begin{array}{c}\text { Complejo } \\
\text { Callialasporites }\end{array}$ & $\begin{array}{l}\text { Callialasporites dampieri (Balme) Dev } 1961 \\
\text { Callialasporites microvelatus Schulz } 1966 \\
\text { Callialasporites segmentatus (Balme) Srivastava } 1963 \\
\text { Callialasporites trilobatus (Balme) Dev } 1961 \\
\text { Callialasporites sp. cf. C. trilobatus (Balme) Dev } 1961 \\
\text { Callialasporites turbatus (Balme) Schulz } 1967 \\
\text { Callialasporites spp. }\end{array}$ & 7 & $\begin{array}{l}2,0 \\
0,3 \\
1,7 \\
0,3 \\
2,7\end{array}$ & 10,3 & $\begin{array}{l}4,4 \\
1,4 \\
1,0\end{array}$ \\
\hline Araucariaceae & $\begin{array}{l}\text { Araucariacites australis Cookson } 1947 \\
\text { Araucariacites fissus Reiser y Williams } 1969 \\
\text { Araucariacites pergranulatus Volkheimer } 1968 \\
\text { Balmeiopsis limbatus (Balme) Archangelsky } 1977 \\
\text { Inaperturopollenites indicus Srivastava } 1966 \\
\text { Inaperturopollenites microgranulatus Volkheimer } 1972\end{array}$ & 1,9 & 1,0 & 12,6 & $\begin{array}{c}2,5 \\
* \\
3,3 \\
1,3 \\
2,7 \\
3,0\end{array}$ \\
\hline Podocarpaceae & $\begin{array}{l}\text { Microcachryidites castellanosii Menéndez } 1968 \\
\text { Podocarpidites ellipticus Cookson } 1947 \\
\text { Podocarpidites verrucosus Volkheimer } 1972 \\
\text { Podocarpáceas indeterminadas }\end{array}$ & 3,3 & $\begin{array}{l}6,9 \\
0,3 \\
0,7 \\
2,3\end{array}$ & & $\begin{array}{l}0,3 \\
3,8\end{array}$ \\
\hline Taxodiaceae & $\begin{array}{l}\text { Cerebropollenites sp. cf. C. macroverrucosus (Thiergart) Schulz } 1967 \\
\text { Perinopollenites? elatoides Couper } 1958\end{array}$ & & & 0,3 & 0,3 \\
\hline & Peroaletes rugosus McKellar 1974 & 0,3 & 0,3 & & \\
\hline Pteridospermales & $\begin{array}{l}\text { Alisporites lowoodensis Jersey } 1963 \\
\text { Alisporites similis (Balme) Dettman } 1963 \\
\text { Vitreisporites pallidus (Reissinger) Nilsson } 1958\end{array}$ & 5,1 & $\begin{array}{l}0,3 \\
1,0 \\
3,8\end{array}$ & 6,0 & $\begin{array}{l}2,7 \\
3,4\end{array}$ \\
\hline Cycad./Bennet./Gingk. & $\begin{array}{l}\text { Cycadopites adjectus (de Jersey) Volkheimer y Quattrocchio } 1975 \\
\text { Cycadopites sp. }\end{array}$ & 0,7 & 0,7 & 0,7 & 0,7 \\
\hline Esporas & $\begin{array}{l}\text { Antulsporites saevus (Balme) Archangelsky y Gamerro } 1966 \\
\text { cf. Conbaculatisporites mesozoicus Klaus } 1960 \\
\text { Concavisporites semiangulatus Menéndez } 1968 \\
\text { Deltoidospora minor (Couper) Pocock } 1970\end{array}$ & 8,5 & $\begin{array}{l}0,3 \\
0,3 \\
0,3 \\
2,4\end{array}$ & 5,6 & 0,3 \\
\hline
\end{tabular}


Tabla 1. (Continuación).

\begin{tabular}{|c|c|c|c|c|c|}
\hline Grupos Supragenéricos & Taxones & & & & \\
\hline Esporas & $\begin{array}{l}\text { Deltoidospora sp. } \\
\text { Duplexisporites sp. } \\
\text { Gemmatriletes sp. cf. G. covuncoensis Volkheimer y Moroni } 1981 \\
\text { Granulatisporites sp. A (en Volkheimer 1968) } \\
\text { Granulatisporites sp. C (en Volkheimer 1972) } \\
\text { Gn. et. sp. indet. } \\
\text { Interulobites lajensis Martínez } 2000 \\
\text { Ischyosporites sp. cf. I. sp. C (en Volkheimer 1972) } \\
\text { Klukisporites variegatus Couper 1958 } \\
\text { Klukisporites sp. cf. K. scaberis (Cookson y Dettmann) Dettmann } 1963 \\
\text { Klukisporites labiatus (Volkheimer) Baldoni y Archangelsky } 1983 \\
\text { Leiotriletes sp. } \\
\text { Matonisporites sp. } \\
\text { Nevesisporites sp. cf. N. radiatus (Chlonova) Srivastava } 1972 \\
\text { Osmundacidites sp. cf. O. wellmanii Couper } 1953 \\
\text { Pilosisporites sp. } 2 \text { (en Martínez, Quattrocchio y Sarjeant 2001) } \\
\text { Retitriletes spp. } \\
\text { Staplinisporites caminus (Balme) Pocock } 1962 \\
\text { Stereisporites antiquasporites (Wilson y Webster) Dettmann } 1963 \\
\text { cf. Varirugosisporites mutabilis Döring 1965 }\end{array}$ & 8,5 & $\begin{array}{l}0,3 \\
1,8 \\
0,3 \\
0,3\end{array}$ & 5,6 & $\begin{array}{l}0,3 \\
0,3 \\
0,7\end{array}$ \\
\hline Chlorophyta & Botryococcus spp. & & * & & \\
\hline Prasinophyceae & Tasmanites sp. & 0,3 & 0,3 & & \\
\hline Zygnemaphyceae & $\begin{array}{l}\text { Mougeotia sp. } \\
\text { Ovoidites sp. }\end{array}$ & 0,8 & $\begin{array}{l}0,3 \\
0,5\end{array}$ & & \\
\hline Acritarcos & $\begin{array}{l}\text { Leiosphaeridia sp. cf. L. hyalina (Deflandre) Downie } 1957 \\
\text { Leiosphaeridia spp. } \\
\text { Acritarco indet. } \\
\text { Michrystridium brevispinosum (Sarjeant) Sarjeant y Stancliffe } 1994 \\
\text { Michrystridium echinoides Valensi } 1953 \\
\text { Michrystridium recurvatum forma recurvatum Valensi } 1953 \\
\text { Michrystridium spp. }\end{array}$ & 2,7 & $\begin{array}{l}0,3 \\
0,6 \\
0,9 \\
0,9\end{array}$ & 3,0 & $\begin{array}{l}0,66 \\
1,35\end{array}$ \\
\hline Dinoquistes & $\begin{array}{l}\text { Acanthaulax downiei (Sarjeant) Sarjeant } 1976 \\
\text { Lithodinia deflandrei Sarjeant } 1968 \\
\text { Eschariphaeridia pocockii (Sarjeant) Erkmen y Sarjeant } 1980\end{array}$ & & & 2,8 & $\begin{array}{l}1,2 \\
0,3 \\
0,7\end{array}$ \\
\hline
\end{tabular}

* Presencia

forma la palinoflora continental de la Formación Helby et al., 1987, para el Tithoniano de Australia en Vaca Muerta. Similar situación fue mencionada por la Zona de Oppel de Retitriletes watherooensis (Back- 


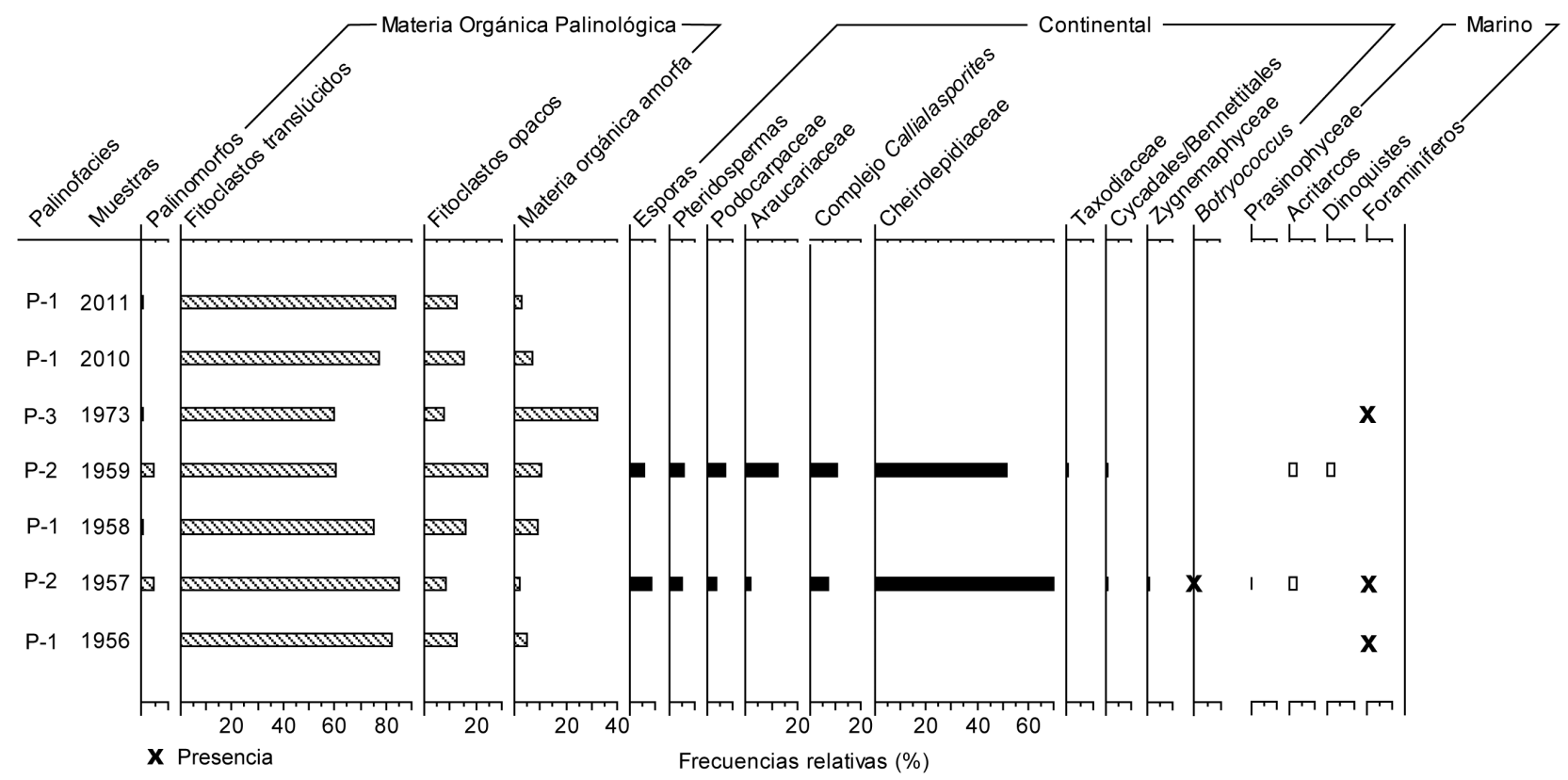

Figura 7. Representación de las palinofacies y distribución de palinomorfos y foraminíferos según frecuencias relativas / representation of the palynofacies and distribution of the palynomorphs and foraminifers according to relative frequencies.

house, 1978, modificada por Helby et al., 1987). En la palinoflora identificada en este trabajo, el complejo Callialasporites varía entre 7 y $10,3 \%$, alcanzando Microcacrhyidites valores máximos de tan sólo 0,3\% (tabla 1). Esto podría sugerir un contexto palinoestratigráfico previo a la Zona de Microcachryidites antarcticus para la presente asociación. Dentro del complejo Callialasporites es también destacable el porcentaje con que se presenta Calilalasporites trilobatus, $4,4 \%$, ya que esta especie ocurre siempre en muy bajas proporciones en diferentes asociaciones de formaciones jurásicas de la Cuenca Neuquina a partir del Caloviano medio. Previo a estos registros, en el Grupo Cuyo, si bien el complejo Callialasporites representa hasta un $17 \%$ de la asociaciones identificadas (Martínez et al., 2008), C. trilobatus es un taxón extremadamente raro cuya presencia es generalmente menor al 1\% (Formación Los Molles, Martínez et al., 2005b) alcanzando excepcionalmente valores máximos de 1,2\% en la Formación Lajas (García et al., 2006) y de 1,4\% en la Formación "Challacó" (= Bosque Petrificado) (Martínez et al., 2005a). Filatoff (1975) registró para el Jurásico de la Cuenca de Perth, Australia, un incremento en el porcentaje de C. trilobatus en la parte alta de la Zona de Oppel de Contignisporites cooksoniae Filatoff (1975), modificada por Helby et al. (1987), que se extiende desde el Bathoniano medio hasta el Caloviano medio temprano. Este incremento se mantiene en toda la Zona de Oppel de Murospora florida Filatoff (1975), modificada por Helby et al. (1987) (Caloviano medio tardío-Kimmeridgiano). El com- portamiento observado en la distribución del complejo Callialalasporites vs. Microcacrhyidites sumado al incremento en la frecuencia relativa de C. trilobatus en el presente trabajo, refuerzan la hipótesis de una asociación posterior al Caloviano medio y previa al Tithoniano.

Por otra parte, el registro de esporomorfos pertenecientes a la Zona de Stereisporites pandoi (Oxfordiano tardío, Quattrocchio et al., 1996): Gemmatriletes sp. cf. G. covuncoensis, Stereisporites antiquasporites y Microcachryidites castellanosii entre otros, permite sugerir una edad máxima no más antigua que el Oxfordiano tardío. Es importante resaltar aquí la presencia en la asociación palinoflorística de un taxón no registrado hasta el momento en nuestro país antes del Thitoniano tardío, Balmeiopsis limbatus (Quattrocchio et al., 2003), muy común en las floras cretácicas argentinas, si bien a nivel mundial ha sido reconocido ya en el Sinemuriano (Srivastava, 1987).

Si bien los dinoquistes son escasos y poco diagnósticos, merece destacarse la presencia de algunos taxones como Lithodinia deflandrei, mencionada mundialmente desde el Batoniano medio hasta el Kimmeridgiano (Bujak y Williams, 1977; Riding, 1982) pero no registrado anteriormente en Argentina, o la presencia de Acanthaulax downiei que si bien posee un registro global que se inicia en el Oxfordiano (Stancliffe y Sarjeant, 1988) es típica de las secuencias tithonianas del país.

Si bien no son abundantes los palinomorfos que permiten una asignación bioestratgráfica muy preci- 


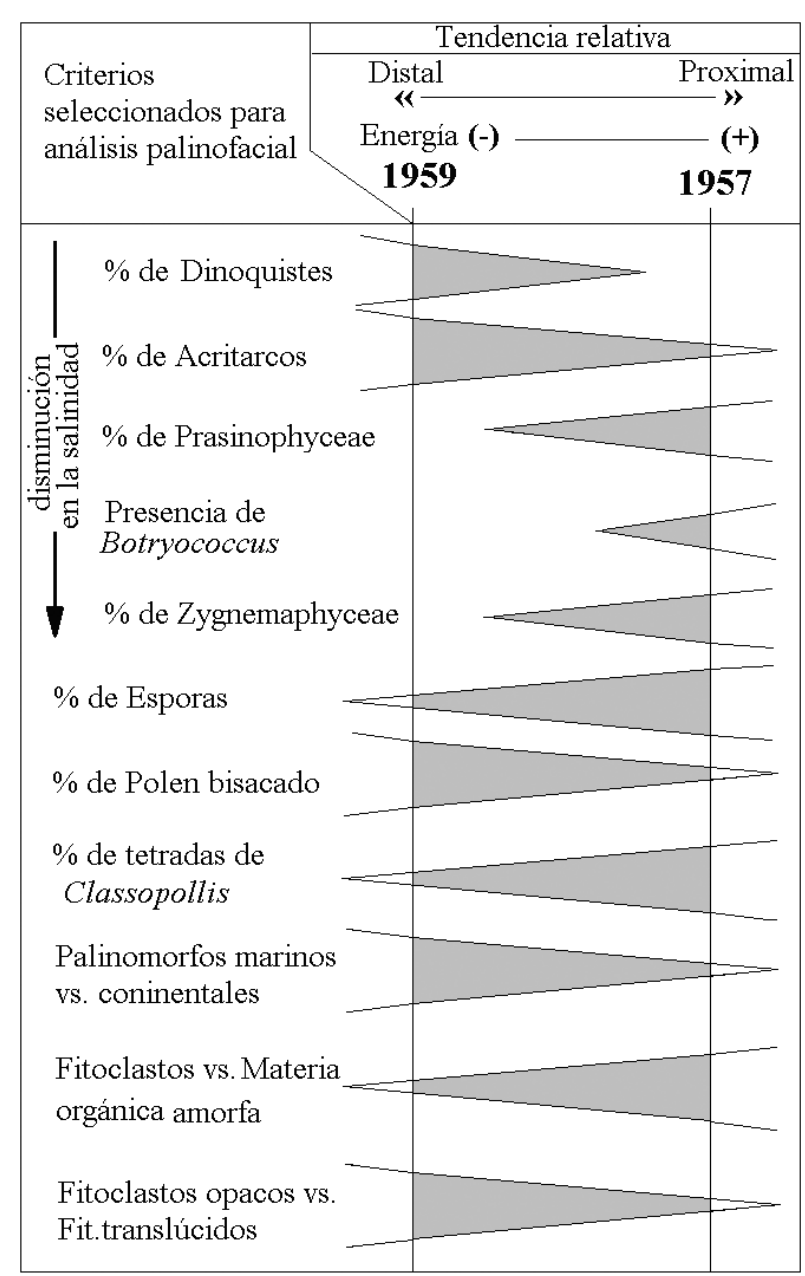

Figura 8. Criterios palinofaciales e interpretaciones paleoambientales relativas aplicadas a la Palinofacies-tipo 2 / palynofacial criteria and relative paleoenvironmental interpretations applied to Palynofacies-type 2.

sa, la identificación de taxones típicos de unidades calovianas y tithonianas muestra el marcado carácter transicional de la presente asociación. Dado que estos depósitos infrayacen en contacto discordante a los depósitos conglomerádicos de la Formación Tordillo (Kimmeridgiano), se propone como edad más probable para la palinoflora estudiada el rango Oxfordiano tardío-Kimmeridgiano temprano.

\section{Discusión acerca del origen y extensión tem- poral de la Formación Lotena}

La denominación de Formación Lotena (Loteno Formation) fue propuesta originalmente por Weaver (1931) para designar a los conglomerados aflorantes en las inmediaciones del cerro Lotena, en el ámbito de la Dorsal de Huincul, Cuenca Neuquina. Esta denominación fue posteriormente extendida al sur de la cuenca (Herrero Ducloux, 1946) para designar a los depósitos clásticos mayormente rojizos con fauna marina del Caloviano localizados entre los niveles marinos de la Formación Lajas (Grupo Cuyo) y la transgresión del Tithoniano representada por Formación Vaca Muerta, ubicada por encima de la denominada "discordancia oxfordiana". Un importante avance para el conocimiento de la unidad se produjo a partir del trabajo de Dellapé et al. (1978), quienes por primera vez caracterizaron, en la localidad del puente de la Ruta Nacional 40 sobre el Arroyo Picún Leufú, a un ciclo marino asignado al Caloviano medio, el cual sería claramente diferenciable de las capas rojas (Formación Challacó) subyacentes del "Cuyano" (=Grupo Cuyo). No obstante, el concepto actual de la unidad fue establecido por Gulisano et al. (1984), quienes reasignaron los conglomerados del Cerro Lotena al Grupo Cuyo, reconociendo a la Formación Lotena como depósitos clásticos marinos a continentales localizados dentro de un importante ciclo sedimentario denominado Loteniano-Chacayano (=Grupo Lotena), ubicado entre las discordancias Intracaloviana e Intramálmica. Gulisano et al. (1984) reconocieron dentro de este ciclo tres secuencias depositacionales mayores, las cuales denominaron como L1, L2 y L3.

De este modo, la Formación Lotena correspondería a depósitos clásticos (areniscas, limolitas grises a verdosas y conglomerados subordinados) acumulados en un medio marino a litoral dentro del intervalo Caloviano medio-Oxfordiano medio. En los afloramientos localizados al norte de la Dorsal de Huincul, el límite superior de la Formación Lotena está caracterizado por un rápido pasaje a una potente sucesión de carbonatos asignados a la Formación La Manga (Stipanicic, 1965), los cuales contienen fauna del Oxfordiano temprano a medio. No obstante, el límite superior de la unidad en los afloramientos localizados al sur de la Dorsal de Huincul no es tan claro, ya que no se han reconocido estos depósitos calcáreos y/o evaporíticos. Muy por el contrario, la Formación Lotena aflorante en la localidad de Estancia María Juana (figura 2) se dispone por debajo de conglomerados y lutitas rojas estériles asignados a la Formación Fortín $1^{\circ}$ de Mayo (Gulisano et al., 1984) e interpretadas por su posición en la secuencia como Oxfordiano tardío. Consecuentemente, los datos proporcionados en este trabajo permiten por primera vez determinar la extensión temporal de la Formación Lotena en el sector sur de la Cuenca Neuquina. La presencia de una asociación palinoflorística con las características mencionadas en párrafos anteriores, que permite sugerir para los niveles estratigraficamente más altos de la Formación Lotena una edad no más antigua que Oxfordiano tardío Kimmeridgiano temprano, es importante ya que re- 
fuerza la hipótesis de una desconexión física entre la Cuenca Neuquina y la Subcuenca Picún Leufú durante el Bajociano tardío-Caloviano (Zavala y González, 2001), pudiendo la misma extenderse temporalmente a edades tan jóvenes como el Kimmeridgiano. Esta desconexión podría estar relacionada con una actividad tectónica temprana en el área de la Dorsal de Huincul (Zavala y González 2001). En consecuencia, es posible afirmar que en el sector sur de la cuenca (Subcuenca Picún Leufú) la conexión marina abierta continuó al menos hasta fines del Oxfordiano tardío-Kimmeridgiano temprano, con la acumulación de facies clásticas marinas asignables, por litología y posición en secuencia, a la Formación Lotena. La edad de la Formación Lotena en el sector sur de la cuenca podría abarcar desde el Caloviano medio hasta el Oxfordiano tardío- Kimmeridgiano temprano, siendo de este modo en parte equivalente a la Formación Fortín $1^{\circ}$ de Mayo. Las facies de carbonatos y evaporitas (formaciones La Manga y Auquilco) reconocidos en el centro de cuenca no se habrían acumulado al sur de la Dorsal de Huincul.

\section{Consideraciones paleoambientales}

El análisis conjunto de las litofacies y las palinofacies permite reconstruir las condiciones paleoambientales imperantes al momento de la depositación de la unidad en análisis. El arreglo de facies sugiere un medio marino de plataforma a litoral, localizado por debajo del tren de olas. La presencia de estructuras diagnósticas del accionar de mareas (tidal bundles; Terwindt, 1981) refuerza la interpretación de una vinculación marina. El predominio de fitoclastos translúcidos no-bioestructurados relacionados con un origen fluvio-deltaico (Tyson, 1995) y esporomorfos (granos de polen y esporas), sumado a la presencia en la palinoflora de palinomorfos de origen marino (dinoquistes proximados y algas prasinofíceas) sugiere un ambiente marinomarginal, próximo al área de aporte continental y de moderada energía. En la figura 8 se muestran los criterios seleccionados para el análisis palinofacial de la Palinofacies-tipo 2 (muestras 1957 y 1959), los cuales pueden ser interpretados en términos de tendencias próximo-distales relativas al área de aporte terrígeno. En este contexto paleoambiental, la muestra 1957 sugiere condiciones relativamente más proximales al área de aporte continental que la muestra 1959. Si bien el microplancton de pared orgánica es escaso, la distribución del mismo permite confirmar esta tendencia, ya que en la muestra 1957 se observa una diversidad de formas desde dulceacuícolas hasta marinas, sugiriendo descarga fluvial en el ambiente marino.

AMEGHINIANA 47 (4), 2010
Asociaciones jurásicas de foraminíferos presentando baja diversidad, con ausencia de formas calcáreas y dominadas por Ammodiscus han sido consideradas indicativas de condiciones marino-marginales, hasta lagunas litorales, en parte hiposalinas. Nagy y Johansen (1991) las han relacionado con ambientes de planicies deltaicas; asimismo Nagy y Berge (2008) las han considerado como evidencia de ambientes de bahías extensas poco profundos, con salinidad disminuida debido al aporte de agua dulce de carácter fluvial.

Nagy (1992) reunió las conchillas uniloculares comprimidas enroscadas tales como Ammodiscus en su morfogrupo 4-a, asignándole hábito libre epifaunal (incluso viviendo sobre plantas acuáticas) y detritívoros (herbívoros) activos. A similares conclusiones arribó Tyszka (1994) quien estudió los foraminíferos del Jurásico de los Cárpatos polacos e incluyó las conchillas uniloculares enroscadas comprimidas en su morfogrupo A-3 y, más recientemente, Reolid et al. (2008) y Nagy et al. (2009) reunieron en su morfogrupo E a aquellas recuperadas en el Jurásico Tardío del sur de España y de Spitsbergen, respectivamente. En algunas conchillas aquí estudiadas, se observa una pequeña porción inicial con un enroscamiento irregular (streptospiral?) del tipo del género Glomospirella; se ha asociado el cambio en el enroscamiento de las conchillas con condiciones ambientales adversas o cambiantes (Chamney, 1976). La presencia de conchillas con contorno irregular estaría reflejando su hábito adherente a un sustrato duro, lo que indicaría algunos episodios de cierta energía (Sturrock y Murray, 1981).

\section{Conclusiones}

Esta contribución permite por primera vez determinar la extensión temporal de la Formación Lotena en el sector sur de la Cuenca Neuquina (Subcuenca Picún Leufú). El marcado carácter transicional de la asociación palinoflorística identificada en los niveles estudiados, en la cual se destacan algunos taxones presentes en nuestro país a partir del Jurásico Tardío como Acanthaulax downiei y Balmeiopsis limbatus, junto con el registro de esporomorfos pertenecientes a la Zona de Stereisporites pandoi Quattrocchio et al., 1996, del Oxfordiano tardío, y las relaciones de campo (depósitos que infrayacen en contacto discordante a los niveles conglomerádicos kimmeridgianos de la Formación Tordillo), permiten sugerir el lapso Oxfordiano tardío-Kimmeridgiano temprano, como edad más probable para la palinoflora estudiada.

$\mathrm{El}$ arreglo de facies sedimentarias, junto a los microfósiles marinos (microplancton de pared orgánica y foraminíferos), permiten inferir la presencia de 
condiciones marinas en este sector de la cuenca hasta fines del Oxfordiano-principios del Kimmeridgiano, reforzando la hipótesis de una desconexión física entre el norte y sur de dorsal de Huincul. Las facies de carbonatos y evaporitas (formaciones La Manga y Auquilco) reconocidos en el centro de cuenca no se habrían acumulado en el sector sur.

El estudio interdisciplinario de los tres proxy-data (análisis sedimentológico y estratigráfico, palinofacial y micropaleontológico) sugiere un ambiente marino marginal, sometido a cambios en la salinidad por el influjo de agua dulce, próximo al área de aporte terrígeno y de moderada energía.

\section{Agradecimientos}

Los autores desean expresar su agradecimiento al Consejo Nacional de Investigaciones Científicas y Técnicas (CONICET) PIP 5297 y la Secretaría General de Ciencia y Tecnología de la Universidad Nacional del Sur (SeGCyT), PICT 24/H083 por el apoyo económico brindado, así como también a los editores y a M.B. Prámparo, E.G. Ottone y A.M. Zavattieri por las sugerencias recibidas oportunamente en calidad de árbitros. A S. Candel por su colaboración en el procesamiento de muestras y confección de gráficos.

\section{Bibliografía}

Abbink, O.A. 1998. Palynological investigations in the Jurassic of the North Sea region. Laboratory of Palaeobotany and Palynology, Contributions Series 8: 192 pp.

Alley, N.F., Krieg, G.W. y Callen, R.A. 1996. Early Tertiary Eyre Formation, Lower Nelly Creek Southern lake Eyre Basin, Australia: Palynological dating of macroflora and silcrete and paleoclimatic interpretations. Australian Journal of Earth Sciences 43: 71-84.

Archangelsky, S. 1977. Balmeiopsis, nuevo nombre genérico para el palinomorfo Inaperturopollenites limbatus Balme 1957. Ameghiniana 14: 122-126.

Archangelsky, S. y Gamerro, J.C. 1966. Estudio palinológico de la Formación Baqueró (Cretácico), Provincia de Santa Cruz II. Ameghiniana 4: 201-209.

Archangelsky, A. y Llorens, M. 2003. Palinología de la Formación Kachaike, Cretácico Inferior de la Cuenca Austral, provincia de Santa Cruz. I. Esporas lisas y cinguladas. Ameghiniana 40: 71-80.

Archangelsky, A. y Llorens, M. 2005. Palinología de la Formación Kachaike, Cretácico Inferior de la Cuenca Austral, provincia de Santa Cruz. II. Esporas. Ameghiniana 42: 311-328.

Archangelsky, S. y Seiler, J. 1980. Algunos resultados palnológicos de la perforación UN OIL OSL, del SO de la Provincia del Chubut, República Argentina. $2^{\circ}$ Congreso Argentino de Paleontología y Bioestratigrafía y $1^{\circ}$ Congreso Latinoamericano de Paleontología (Buenos Aires, 1978), Actas 5: 215-225.

Archangelsky, S., Baldoni, A., Gamerro, J.C. y Seiler, J. 1984. Stratigraphic Palynology of the Cretaceous in Southern Argentina. III. Distribution of species and conclusions. Ameghiniana 21: 15-33.

Backhouse, J. 1978. Palynological zonation of the Late Jurassic and Early Cretaceous sediments of the Yarragadee Formation, central Perth Basin, Western Australia. Report Geological Survey of Western Australia 7: 1-53.

Baldoni, A.M. y Archangelsky, S. 1983. Palinología de la Forma- ción Springhill (Cretácico Inferior), Subsuelo de Argentina y Chile Austral. Revista Española de Micropaleontología 15: 47- 101.

Baldoni, A. y Batten, D.J. 1991. Megaspores from the Lower Cretaceous Kachaike Formation, Santa Cruz Province, Argentina. Neues Jahrbuch fur Geologie und Paläontologie, Abhandlungen 182: 377-393.

Balme, B.E. 1957. Spores and pollen grains from the Mesozoic of Western Australia. Commonwealth Scientific and Industrial Research Organization, Coal Research Section, Technical Communication 25: $48 \mathrm{pp}$.

Boltovskoy, E. 1965. Los foraminíferos recientes (Biología, métodos de estudio, aplicación oceanográfica). EUDEBA, Editorial Universitaria, Buenos Aires, $510 \mathrm{pp}$.

Bornemann, L.G. 1874. Ueber die Foraminiferengattung Involutiva. Zeitschrift der Deutschen Geologischen Gesellschaft 26: 702-749.

Bujak, J.P y Williams, G.L. 1977. Jurassic palynostratigraphy of offshore Eastern Canada. Stratigraphic Micropaleontology of Atlantic Basin and Borderlands: 321-339.

Burger, D. 1965. Some new species of Classopollis from the Jurassic of the Netherlands. Leidse Geologische Mededelingen 33: 63-69.

Chamney, T.P. 1976. Foraminiferal morphogroup symbol for paleoenvironmental interpretation of drill cutting samples: Arctic America, Albian continental margin. Special Publications-Maritime Sediments 1B: 585-624.

Cookson, I.C. 1947. Plant microfossils from the lignites of the Kerguelen Archipelago. British and New Zealand Antarctic Research Expedition, 1929-1931, Reports, series A, 2: 129-142.

Couper, R.A. 1953. Upper Mesozoic and Cenozoic spores and pollen grains from New Zealand. New Zealand Geological Survey, Paleontological Bulletin 22: 1-77.

Couper, R.A. 1958. British Mesozoic microspores and pollen grains. A systematic and stratigraphie study. Palaeontographica Abteilung B, 103: 75-179, Stuttgart.

Deák, M.H. 1962. Deux nouveaux genres de spore de la série d'argiles et de marnes aptiennes. Bulletin de la Société Géologique de Hongrie 92: 230-235.

de Jersey, N.J. 1963. Jurassic spores and pollen grains from the Marburg Sandstone. Geological Survey of Queensland (313): 15 pp.

de Jersey, N.J. 1971. Early Jurassic miospores from the Helidon Sandstone. Geological Survey of Queensland, Publication 351, Palaeontological Papers 25, 49 pp.

Dellapé, D., Pando, G. y Volkheimer, W. 1978. Estratigrafía y palinología de las Formaciones Mulichinco, Agrio y Grupo de La Amarga, al sur de Zapala (Provincia del Neuquén). $7^{\circ}$ Congreso Geológico Argentino (Neuquén), Actas 1: 593-607.

Dettmann, M.E. 1963. Upper Mesozoic microfloras from southeastern Australia. Proceedings of the Royal Society of Victoria 77: $1-148$.

Dev, S. 1961. The fossil flora of Jabalpur Series- 3. Spores and pollen grains. The Palaeobotanist 8: 43-56.

Dörhöfer, G. 1977. Palynologie und Stratigraphie der BückebergFormation (Berriasium-Valanginium). Geologisches Jahrbuch 42: 3-122.

Döring, H. 1965. Die sporenpaläontologische Gliederung des Wealden in Westmecklenburg. Geologie 14: 1-118.

Downie, C. 1957. Microplankton from the Kimmeridge Clay. Quarterly Journal of the Geological Society of London 112: 413-434.

Erkmen, U. y Sarjeant, W.A.S. 1980. Dinoflagellate cysts, acritarchs and tasmanitids from the uppermost Callovian of England and Scotland: with a reconsideration of the "Xanthidium pilosum " problem. Geobios 13: 45-99.

Filatoff, J. 1975. Jurassic Palynology of the Perth Basin, Western Australia. Palaeontographica, Abteilung B 154: 1-113.

García, V.M., Quattrocchio. M.E., Zavala, C.A. y Martínez, M.A. 2006. Palinofacies, paleoambientes y paleoclima del Grupo Cuyo (Jurásico Medio) en la Sierra del Chacaico, Cuenca Neuquina, Argentina. Revista Española de Micropaleontología 38: 269-288.

Gocht, H. 1975. Morphologie und Wandstruktur von Lithodinia ju- 
rassica Eisenack 1935 (Dinoflagellata, Oberjura). Neues Jahrbuch fur Geologie und Paläontologie, Monatsheft 6: 343-359.

Gocht, H. 1976. Hystrichosphaeropsis quasicribrata (O. Wetzel), ein Dinoflagellat aus dem Maastricht Nordeuropas (mit einem nomenklatorischen Nachtrag zur Gattung Lithodinia Eis.) Neues Jahrbuch fur Geologie und Paläontologie, Monatsheft 6: 321-336.

Groeber, P. 1946. Observaciones geológicas a lo largo del Meridiano 70. Hoja Chos Malal. Revista de la Asociación Geológica Argentina 1: 117-208.

Groeber, P. 1951. La Alta Cordillera entre las latitudes $34^{\circ}$ y 29³0'. Revista del Museo Argentino de Ciencias Naturales Bernardino Rivadavia, Ciencias Geológicas 1: 235-352.

Groot, J.J. y Groot, C.R. 1962. Plant microfossils from Aptian, Albian and Cenomanian deposits of Portugal. Comunicações dos Serviços Geológicos de Portugal 46: 133-171.

Gulisano, C.A. 1981. El Ciclo Cuyano en el norte de Neuquén y sur de Mendoza. $8^{\circ}$ Congreso Geológico Argentino (San Luis) Actas 3: 579-592.

Gulisano, C.A. y Gutiérrez Pleimling, A.R. 1995. Guía de campo. El Jurásico de la Cuenca Neuquina, b) Provincia de Mendoza. Asociación Geológica Argentina, Serie E 3: 1-103.

Gulisano, C.A., Gutiérrez Pleimling, A.R. y Digregorio, R.E. 1984 Esquema estratigráfico de la secuencia Jurásica del oeste de la provincia del Neuquén. $9^{\circ}$ Congreso Geológico Argentino (Bariloche), Actas 1: 236-259

Helby, R., Morgan, R. y Patridge, A.D. 1987. A palynological zonation of the Australian Mesozoic. Memoir of the Asociation of Australasian Palaeontologists 4: 1-94.

Herrero Ducloux, A. 1946. Contribución al conocimiento geológico del Neuquén extrandino. Boletín de Informaciones Petroleras 23: 1-39.

Klaus, W. 1960. Sporen der Karnischen Stufe der ostalpinen Trias. Jahrbuch der Geologischen Bundesanstalt 5: 107-184.

Krutzsch, W. 1959. Sporen- und Pollengruppen aus der Oberkreide und dem Tertiär Mitteleuropas und ihre stratigraphische Verteilung. Zeitschrift fur angewandte Geologie 3: 519-548.

Lalicker, C.G. 1950. Foraminifera of the Ellis Group, Jurassic, at the type locality. University of Kansas Paleontological Contributions, Protozoa, Article 2: 3-20.

Legarreta, L. y Gulisano, C.A. 1989. Análisis estratigráfico secuencial de la Cuenca Neuquina (Triásico superior-Terciario). En: G. Chebli y L. Spalletti (eds.), Cuencas Sedimentarias Argentinas, Facultad de Ciencias Naturales, Universidad Nacional de Tucumán, Serie Correlación Geológica 6: 221-243, Tucumán.

Legarreta, L. y Uliana, M.A. 1999. El Jurásico y Cretácico de la Cordillera Principal y la Cuenca Neuquina. En: R. Caminos (ed.), Geología Argentina. Servicio Geológico Minero Argentino, Anales 29: 399-416.

Loeblich, Jr.A.R. y Tappan, H. 1988. Foraminiferal genera and their classification. Van Nostrand Reinhold Company, Nueva York, $970 \mathrm{pp}$

Martínez, M.A. 2000. Nueva especie de Interulobites, espora trilete del Jurásico Medio de la Cuenca Neuquina, Argentina. Revista Española de Paleontología 15: 165-170.

Martínez, M.A. 2002. Palynological zonation of the Lajas Formation (Middle Jurassic) of the Neuquén Basin, Argentina. Ameghiniana 39: 221-240.

Martínez, M.A., Quattrocchio, M.E., Sarjeant, W.A.S. 2001. Análisis palinoestratigráfico de la Formación Lajas, Jurásico Medio de la Cuenca Neuquina, Argentina. Revista Española de Micropaleontología 33: 31-58.

Martínez, M.A., García, V.M. y Quattrocchio, M.E. 2005a. Análisis palinofacial de la Formación Challacó, Jurásico Medio de la cuenca Neuquina, Argentina. Revista Española de Micropaleontología 37: 241-258.

Martínez, M.A., Quattrocchio, M.E. y Prámparo, M.B. 2005b. Análisis palinológico de la Formación Los Molles, Grupo Cuyo, Jurásico Medio de la Cuenca Neuquina, Argentina. Ameghiniana 42: 67-92.

Martínez, M.A., Prámparo, M.B., Quattrocchio, M.E. y Zavala,
C.A. 2008. Depositional environments and hydrocarbon potential of the Middle Jurassic Los Molles Formation, Neuquén Basin, Argentina: palynofacies and organic geochemical data. Revista Geológica de Chile 32: 279-305.

McKellar, J.A. 1974. Jurassic miospores from the upper Evergreen Formation, Hutton Sandstone, and basal Injune Creek Group, North-Eastern Surat Basin. Geological Survey of Queensland, Publication 361, Palaeontological Papers 35, 50 pp.

Menéndez, C.A. 1968. Estudio palinológico del Jurásico Medio de Picún Leufú, Neuquén. Ameghiniana 5: 379-405.

Mpodozis, C. y Ramos, V.A. 1989. The Andes of Chile and Argentina. En: G.E. Ericksen, M.T. Csañas Pinochet y A. Reinemud (eds.), Geology of the Andes and its relations to Hydrocarbon and Mineral Resources. Circumpacific Council for Energy and Mineral Resources, Earth Sciences Series 11: 59-90.

Nagy, J. 1992. Environmental significance of foraminiferal morphogroups in Jurassic North Sea deltas. Palaeogeography, Palaeoclimatology, Palaeoecology 95: 111-134.

Nagy, J. y Berge, S. H. 2008. Micropalaeontological evidence of brackish water conditions during deposition of the Knorringfjellet Formation, Late Triassic-Early JUrassic, Spitsbergen. Polar Research 27: 413-427.

Nagy, J. y Johansen, H.O. 1991. Delta-influenced foraminiferal assemblages from the Jurassic (Toarcian-Bajocian) of the northern North Sea. Micropaleontology 37: 1-40.

Nagy, J., Reolid, M. y Rodríguez-Tovar, F. J. 2009. Foraminiferal morphogroups in dysoxic shelf deposits from the Jurassic of Spitsbergen. Polar Research 28: 214-221.

Nilsson, T. 1958. Über das Vorkommen eines mesozoischen Sapropelgesteins in Schonen. Lunds Universitets Arsskrift 2: 112 pp.

Pierce, R.L. 1961. Lower Upper Cretaceous plant microfossils from Minnesota. Minnesota Geological Survey Bulletin 42: 86 pp.

Playford, G. y Dettmann, M.E. 1965. Rhaeto-Liassic plant microfossils from the Leigh Creek Coal Measures, South Australia. Senckenbergiana Lethaea 46: 127-181.

Pocock, S.A.J. 1962. Microfloral analysis and age determination of strata at the Jurassic-Cretaceous boundary in the western Canada Plains. Palaeontographica, Abteilung B, 111: 1-95.

Pocock, S.A.J. 1970. Palynology of the Jurassic sediments of Western Canada. Part 1. Terrestrial species. Palaeontographica Abteilung B, 130: 12-72.

Pocock, S.A.J. y Jansonius, J. 1961. The pollen genus Classopollis Pflug 1953. Micropaleontology 7: 439-449.

Potonié, R. y Kremp, G. 1954. Die Gattungen der Paläozoichen sporae dispersae und ihre stratigraphie. Geologisches Jahrbuch 69: 111-194.

Prámparo, M.B. 1994. Lower Cretaceous Palynoflora of the La Cantera Formation, San Luis Basin: Correlation with other Cretaceous Palynofloras of Argentina. Cretaceous Research 15: 193-203.

Quattrocchio, M.E., Sarjeant, W.A.S. y Volkheimer, W. 1996. Marine and terrestrial Jurassic microfloras of Neuquén Basin (Argentina): Palynological Zonation. En: A.C. Riccardi (ed.), Advances in Jurassic Research. Transtec Publications, Switzerland. GeoResearch Forum 1-2: 167-178.

Quattrocchio, M.E., Martínez, M.A., García, V.M., y Zavala C.A. 2003. Palinoestratigrafía del Tithoniano-Hauteriviano del centro-oeste de la Cuenca Neuquina, Argentina. Revista Española de Micropaleontología 35: 51-74.

Reiser, R.F., Williams, A.J. 1969. Palynology of the Lower Jurassic sediments of the northern Surat Basin, Queensland. Geological Survey of Queensland publication 339 : $24 \mathrm{pp}$.

Reolid, M., Rodríguez-Tovar, F.J., Nagy, J., Olóriz, F. 2008. Benthic foraminiferal morphogroups of mid to outer shelf environments of the Late Jurassic (Prebetic Zone, Southern Spain): Characterization of biofacies and environmental significance. Palaeogeography, Palaeoclimatology, Palaeoecology 261: 280-299.

Reuss, A.E. 1862. Entwurf einer systematischen Zuzammenstellung der Foraminiferen. Sitzungsberichte der Kaiser-

AMEGHINIANA 47 (4), 2010 
lichen Akademie der Wissenschaften in Wien, MathematischNaturwissenschaftliche Classe (1861) 44: 355-396.

Reyre, Y. 1973. Palynologie du Mésozoique Saharien. Mémories du Muséum National d' Historie Naturell, Paris, Serie C 27: 1-284.

Rich, T.H., Rich, P.V., Wagstaff, B.E., McEwen-Mason, J., Douthitt, C.B. y Gregory, R.T. 1989. Early Cretaceous biota from the Northern side of the Australo-Antarctic rift valley. En: J.A. Crame (eds.) Origins and Evolution of the Antartic Biota. Geological Society, London, Special Publication 47: 121130.

Riding, J. 1982. Jurassic dinocysts from the Warboys Borehole, Cambridgeshire, England. Journal of Micropaleontology 1: 13-18.

Rojo, L.D. y Zavattieri, A.M. 2005. Estudio microflorístico de las formaciones Potrerillos y Cacheuta (Triásico) en el sur del cerro Cacheuta, Mendoza, Argentina. Parte 1. Ameghiniana 42: 320.

Sarjeant, W.A.S. 1961. Microplankton from the Kellaways Rock and Oxford Clay of Yorkshire. Palaeontology 4: 90-118.

Sarjeant, W.A.S. 1976. English Jurassic dinoflagellate cysts and acritarchs: a re-examination of some type and figured specimens. Geoscience and Man 15: 1-24.

Sarjeant, W.A.S. 1982. The dinoflagellate cysts of the Gonyaulacysta Group: a morphological and taxonomic restudy. American Association of Stratigraphic Palynologists Contributions Series 9: 1-81.

Stancliffe, R.P.W. y Sarjeant, W.A.S. 1994. The acritarch genus Veryhachium Deunff 1954, emend, Sarjeant and Stancliffe 1994; a taxonomic restudy and a reassessment of its constituent species. Micropaleontology 40: 223-241.

Schulz, E. 1967. Sporenpaläontologische Untersuchungen ratoliassischer Schichten im Zentralteil des Germanischen Beckens. Paläontologische Abhandlungen B 2: 541-633.

Srivastava, S.K. 1963. Polospores from Jurassic of Rajasthan, India. Nature 198: 1323-1324.

Srivastava, S.K. 1966. Jurassic microflora from Rajasthan, India. Micropaleontology 12: 87-103.

Srivastava, S.K. 1972. Systematic description of some spores from the Edmonton Formation (Maastrichtian), Alberta, Canada. Palaeontographica Abteilung B, 139: 1-46.

Srivastava, S.K. 1987. Jurassic spore-pollen assemblages from Normandy (France) and Germany. Geobios 20: 5-79.

Stancliffe R.P.W. y Sarjeant, W.A.S. 1988. Oxfordian dinoflagellate cysts and provincialism. International Symposium on Jurassic Stratigraphy, 2ND: 763-798.

Stipanicic, P.N. 1965. El Jurásico en Vega de la Veranda (Neuquén), el Oxfordense y el diastrofismo diversiano (AgassizYaila) en la Argentina. Revista de la Asociación Geológica Argentina 20: 403-478.

Sturrock, S. y Murray, J.W. 1981. Comparison of low energy and high-energy marine middle shelf foraminiferal faunas, Celtic Sea and western English Channel. En: J.W. Neale y M.D. Brasier (eds.), Microfossils from Recent and Fossil Shelf Seas. Ellis Horwood Ltd, Chichester: 250-260.

Terquem, O. 1863. Troisième mémoire sur lês foraminifères du Lias des Départements de la Moselle, de la Côte d'Or, du Rhône de la Vienne et du Calvados. Mémoires de l'Académie Impériale de Metz 44: 151-228.

Terwindt, J.H.J., 1981. Origin and sequences of sedimentary structures in inshore mesotidal deposits of the North Sea. International Association of Sedimentology, Special Publication 5: 4-26.

Tyson, R.V. 1995. Sedimentary Organic Matter: Organic Facies and Palynofacies. Chapman and Hall, London, 615 pp.

Tyszka, J. 1994. Response of Middle Jurassic benthic foraminiferal morphogroups to dysoxic/anoxic conditions in the Pieniny Klippen Basin, Polish Carphatians. Palaeogeography, Palaeoclimatology, Palaeoecology 110: 55-81.
Valensi, L. 1953. Microfossiles des silex du Jurassique moyen. Remarques pétrographiques. Mémoires de la Société Géologique de France 68: 1-100.

Vicente, J.C. 2006. Dynamic Paleogeography of the Jurassic Andean Basin: Pattern of regression and general considerations on main features. Revista de la Asociación Geológica Argentina 61: 408-437.

Volkheimer, W. 1968. Esporas y granos de polen del Jurásico de Neuquén (República Argentina). 1. Descripciones sistemáticas. Ameghiniana 5: 333-370.

Volkheimer, W. 1972. Estudio palinológico de un carbón Caloviano de Neuquén. Consideraciones sobre los paleoclimas jurásicos de la Argentina. Revista del Museo de La Plata (Nueva Serie), Sección Paleontología 6: 101-157.

Volkheimer, W. y Moroni, A.M. 1981. Datos palinológicos de la Formación Auquinco, Jurásico Superior de la Cuenca Neuquina, Argentina. $8^{\circ}$ Congreso Geológico Argentino (San Luis), Actas 4: 795-812.

Volkheimer, W. y Papú O.H. 1993. Una microflora del Triásico Superior de la Cuenca de Malargüe, localidad Llantenes, Provincia de Mendoza, Argentina. Ameghiniana 30: 93-100.

Volkheimer, W. y Prámparo, M.B. 1993. Primeros datos palinológicos de la Formación Tordillo (Kimmeridgiano) localidad de Chacay Melehue, Cuenca Neuquina, Argentina. $12^{\circ}$ Congreso Geológico Argentino y $2^{\circ}$ Congreso de Exploración de Hidrocarburos (Mendoza), Actas 2: 327-332.

Volkheimer, W. y Quattrocchio, M.E. 1975. Palinología estratigráfica del Titoniano (Formación Vaca Muerta) en el área de Caichigüe (Cuenca Neuquina). Parte A: especies terrestres. Ameghiniana 12: 193-241.

Volkheimer, W. y Quattrocchio, M.E. 1981. Distribución estratigráfica de los palinomorfos jurásicos y cretácicos en la faja andina y áreas adyacentes de América del Sur Austral con especial consideración de la Cuenca Neuquina. En: W. Volkeimer y E.A. Musacchio (eds.), Cuencas Sedimentarias del Jurásico y Cretácico de América del Sur. Comité Sudamericano del Jurásico y Cretácico 2: 407-444.

Volkheimer, W. y Zavattieri, A.M. 1991. Aratrisporites compositus n. sp. a guide-fossil from the Triassic Cuyo Basin, western Argentina. Neues Jahrbuch fur Geologie und Paläontologie, Monatshefte 9: 564-578.

Wall, J.H. 1960. Jurassic microfaunas of Saskatchewan. Department of Mineral Resources, Petroleum and Natural Gas Branch, Geology Division, Report 53: 1-229.

Weaver, C.E. 1931. Paleontology of the Jurassic and Cretaceous of West-Central Argentina. Memoirs University of Washington 1: 1469.

Zavala, C.A. 2005. Tracking sea bed topography in the Jurassic. The Lotena Group in the Sierra de la Vaca Muerta (Neuquén Basin, Argentina). Geologica Acta 2: 133-145.

Zavala, C.A. y González, R. 2001. Estratigrafía del Grupo Cuyo (Jurásico inferior-medio) en la Sierra de la Vaca Muerta, Cuenca Neuquina. Boletín de Informaciones Petroleras 65: 52-64.

Zavala, C.A., Olivera, D.E., Martínez, M.A. y Ballent, S.C. 2008. Estudio sedimentológico y micropaleontológico de los depósitos clásticos del Oxfordiano en el sur de la Cuenca Neuquina. $17^{\circ}$ Congreso Geológico Argentino (Jujuy) Actas 2: 807-808.

Zippi, P.A. y Bajc, A.F. 1990. Note: Recognition of a Cretaceous Outlier in Northwestern Ontario. Canadian Journal of Earth Science 27: 306-311.

Recibido: 27 de marzo de 2009.

Aceptado: 7 de mayo de 2010. 